\title{
A Multiphase Model Describing Vascular Tumour Growth
}

\author{
CHRISTOPHER J. W. BREWARD \\ Mathematical Institute, \\ 24-29 St Giles, \\ Oxford. \\ OX1 3LB \\ HELEN M. BYRNE \\ School of Mathematical Sciences, \\ University of Nottingham, \\ Nottingham. \\ NG7 2RD \\ CLAIRE E. LEWIS \\ Academic Unit of Pathology, \\ Division of Genomic Medicine, \\ University of Sheffield Medical School, \\ Beech Hill Road, \\ Sheffield. \\ S10 2RX
}

In this paper we present a new model framework for studying vascular tumour growth, in which the blood vessel density is explicitly considered. Our continuum model comprises conservation of mass and momentum equations for the volume fractions of tumour cells, extracellular material and blood vessels. We include the physical mechanisms that we believe to be dominant, namely birth and death of tumour cells, supply and removal of extracellular fluid via the blood and lymph drainage vessels, angiogenesis and blood vessel occlusion. We suppose that the tumour cells move in order to relieve the increase in mechanical stress caused by their proliferation. We show how to reduce the model to a system of coupled partial differential equations for the volume fraction of tumour cells and blood vessels and the phase averaged velocity of the mixture. We consider possible parameter regimes of the resulting model. We solve the equations numerically in these cases, and discuss the resulting behaviour. The model is able to reproduce tumour structure that is found in vivo in certain cases. Our framework can be easily modifed to incorporate the effect of other phases, or to include the effect of drugs.

(c) 2004 Society for Mathematical Biology

\section{Introduction}

The vascular stage of tumour development is characterised by rapid growth, high levels of angiogenesis and co-opting of preexisting vessels (Bicknell et al., 1997). Oxygen, nutrients and water are all supplied internally to the tumour mass via the vasculature, and thus such a tumour can grow much larger than its avascular counterpart, whose growth is diffusion limited. Liquid can also be removed from such tumours through the lymph drainage system. Vascular tumours are dangerous not only because of their own growth, but also because their cells can invade through the tumour vessel walls, travel through the blood stream, and seed secondary tumours (metastases) elsewhere in the body. 
While all vascular tumours are heterogeneous, details of their spatial composition and growth rate vary markedly. For example, some human vascular tumours are irregularly shaped and contain areas of densely packed blood vessels surrounded by viable proliferating tumour cells. Elsewhere in the same tumour, there may be avascular regions devoid of blood vessels in which extensive necrosis (cellular degradation) occurs (Beliën et al., 1999). By contrast, other tumours which have a constant blood vessel density have been seen or reported (Tozer and Lewis, unpublished observations; Bicknell et al. (1997)). Further, tumours implanted beneath the skin of mice remain approximately spherical during growth, in a manner reminiscent of multicell spheroids cultured in vitro. These tumours develop a central necrotic core, surrounded by a region of proliferating cells in which the volume fraction of blood vessels appears to be approximately constant (Brown et al., 2002). The rate at which a tumour's volume increases over time seems to be strongly tumour specific, with growth rates that are linear (for murine tumours (Brown et al., 2002)), quadratic (for a human glioblastoma multiform (Kozin et al., 2001) and for tumours derived from Neuro2a cells (Todo et al., 2001), implanted into the flank of mice), or cubic (for tumours derived from murine lewis lung carcinoma cells (Liao et al., 2000) and for tumours derived from murine breast carcinoma cells (Candido et al, 2001), implanted into the flank of mice) reported in the literature.

The reasons for the presence of the central necrotic region and the outer rim in which the blood vessel density remains constant in murine tumours remain open questions. However, it is well known (Boucher and Jain, 1992) that the interstitial pressure within a tumour is much higher than in normal tissue. Exerting excess pressure on a blood vessel can cause it to buckle and collapse (Boucher and Jain, 1992) thereby halting the supply of oxygen to its microenvironment. This, in turn, can cause the tumour cells supported by the blood vessel to die through lack of oxygen (oxygen-induced necrosis). Thus an area of cellular debris can be generated by a local build-up of pressure. In some cases, death of the tumour cells surrounding the occluded vessel can relieve the pressure and cause the blood vessel to decompress (Griffon-Etienne et al., 1999). Oxygen can then be supplied to the region and any quiescent cells can become proliferating once again. Vascular tumours may, therefore, have significant spatio-temporal inhomogeneity.

A number of attempts have been made to model the growth of vascular tumours. On the microscale, several models based on the Krogh Cylinder model (Krogh, 1919) have been proposed (Baish et al., 1996; Maseide and Rofstad, 2000) whilst Breward et al. (2001) followed the approach of Ward and King (1997). These papers focus on the interactions between a blood vessel supplying oxygen to a region of tissue within a tumour; the latter paper incorporating the effect of blood vessel compliance on the subsequent remodelling of the tumour tissue. On the macroscale, Byrne and Chaplain (1995) studied the distribution of a blood-borne nutrient in a vascularised tumour, where the effects of the vasculature are modelled by introducing a distributed source term into their nutrient equation. Jackson and Byrne (2000) studied how a blood-borne chemotherapeutic drug altered the size of a vascular tumour. In Hahnfield et al. (1999), a simple, spatially averaged model is developed and compared with experimental data. The model couples the growth rate of the tumour volume with changes in the vascular volume and also accounts for changes in the vascular volume caused by anti-angiogenic agents such as endostatin. Orme and Chaplain (1996) formulate a model for a vascular tumour that has two species - tumour cells and blood vessels. They assume that the tumour cells move by diffusion and 'taxis' up gradients of blood vessels. They model the 'high pressure causing pressure collapse' by including a (nonlinear) vessel death term that increases dramatically as the density of the tumour cells increases. They model the region of tissue outside the tumour by defining an external blood vessel profile. They found that, with "realistic" initial conditions, the tumour developed a necrotic core, devoid of blood vessels. The profiles were such that tumour cells existed when there were no blood vessels, and the blood vessel density was monotonic decreasing from the tumour's periphery. They did not, however, quantify 
the speed of growth of the tumour, or account for conservation of mass.

Two-phase models have been successfully used to characterise avascular tumour growth (see Ward and King (1997), Breward et al. (2001)). In these models, one phase always comprises live tumour cells, while the second phase comprises either dead or extracellular material. Two phases are required in the avascular case, since live cells take in volume from the second phase in order to proliferate. To extend such models systematically to describe the behaviour of a vascular tumour, in which the spatio-temporal distribution of blood vessels is key to understanding the growth of the tumour, we must necessarily have three phases.

In this paper we present a mathematical model to describe the growth of a vascular tumour. Our aim is to capture the features of murine tumour growth described earlier, that is, to focus on growth in which we obtain a vascular tumour with a central necrotic core. Our model is a natural extension of the two-phase model presented by Breward et al. (2002) to describe the growth of avascular tumours. Here, we suppose that our tumour consists of three phases, namely tumour cells, blood vessels and extracellular material. The extracellular material consists of extracellular water (required as building blocks for growth of tumour cells), the waste products of cellular decay, extracellular matrix and the lymph drainage system. In essence, the extracellular material consists of everything apart from tumour cells and blood vessels. This is a simplification to minimise the complexity of the model; the aim of this paper is to present the modelling framework. Decomposing the extracellular material into its constituents is a natural extension. We note that stromal cells may also be considered to reside in this component. We assume that our blood vessel phase consists of co-opted vessels and neovasculature formed by tumour angiogenesis, even though in practice, these two types of blood vessels have different properties. The co-opted vessels have a more-developed basement membrane and are thus able to withstand intra-tumour pressure. In contrast, the neovasculature is leaky (Hashizume et al., 2000) and has a badly developed basement membrane. Thus such vessels are more prone to collapse than their co-opted counterparts. As these new vessels become "more mature", their basement membrane becomes more developed and their behaviour more akin to that of the co-opted vessels. In this paper, we shall assume that the blood vessels in the tumour have some "average" properties. (We discuss extending the model to explicitly track the two populations in §5.) Our model will be one-dimensional, and, as such, will not take into account any details of vessel morphology (for example, the vessel width, rate of blood flow, or vessel tortuosity). Further, we do not introduce a separate dependent variable to describe the local nutrient concentration. We shall assume, instead, that the oxygen tension at any point within our tumour is directly proportional to the number of blood vessels there. This assumption has the immediate consequence that our new model will not reduce to a plausible model for avascular tumour growth in the limit of volume fraction of blood vessels going to zero. If we wished to allow our model to reduce to a sensible avascular limit, we would need to track the oxygen tension explicitly. Finally, we note that angiogenesis must be an ongoing process even in areas which are, on the macroscale, well vascularised. This is because, in the absence of angiogenesis but the presence of cell birth, the blood vessel volume fraction would necessarily decrease.

We formulate our one-dimensional mathematical model by applying conservation of mass and momentum to each of the three phases. We explicitly track the pressure within the tumour, and include vessel death if the pressure the cells exert on the vessel exceeds a threshold value. We also include vessel birth (angiogenesis), tumour cell birth and death (the former depending on all three phases, the latter depending on the number of tumour cells and blood vessels present), and the supply of water from the blood vessels and its drainage through the lymph system. We also allow for co-opting of vessels at the tumour's periphery.

Our model differs from those that already exist in the literature because we (i) have a three-phase model, (ii) have an adaptive vasculature whose dynamics are coupled with those of the tumour cells, 
and (iii) include vessel collapse due to excess pressure (explicitly calculating the pressure).

In $\S 2$, we derive the mathematical model that governs the evolution of our vascular tumour. In $\S 3$, we manipulate the model equations to obtain the most tractable system of equations. In $\S 4$ we focus on special cases for which the model equations simplify greatly. In $\S 5$ we draw together our conclusions and suggest model extensions.

\section{Mathematical model}

As noted in the introduction, we formulate a one-dimensional model for a vascular tumour in which the spatial dimension is denoted by $x$ and the tumour's width is given by $l(t)$. We denote the volume fractions of tumour cells, extracellular material, and blood vessels by $\alpha, \beta$ and $\gamma$ respectively. We formulate conservation of mass equations under the assumption that each phase has the same (constant) density ${ }^{\dagger}$. We write

$$
\begin{aligned}
& \frac{\partial \alpha}{\partial t}+\frac{\partial}{\partial x}\left(u_{\alpha} \alpha\right)=q_{\alpha}, \\
& \frac{\partial \beta}{\partial t}+\frac{\partial}{\partial x}\left(u_{\beta} \beta\right)=q_{\beta}, \\
& \frac{\partial \gamma}{\partial t}+\frac{\partial}{\partial x}\left(u_{\gamma} \gamma\right)=q_{\gamma} .
\end{aligned}
$$

In equations (1)-(3), $u_{\alpha}$ is the speed with which the tumour cells move, $u_{\beta}$ the speed with which the extracellular phase moves, $u_{\gamma}$ the speed with which the blood vessel phase moves, $q_{\alpha}$ represents the rate of change of tumour cell volume fraction due to cell birth and death, $q_{\beta}$ represents the rate of change of extracellular material volume fraction due to cell birth and death, vessel death and liquid entering and leaving the tumour, and $q_{\gamma}$ represents the rate of change of blood vessel phase volume fraction due to the generation or occlusion of vessels. We assume that, in any internal control volume, the tumour consists exclusively of these three constituents (i.e. there are no voids, see Ward and King (1997)) and so we set

$$
\alpha+\beta+\gamma=1 .
$$

We further assume that momentum is conserved. Denoting the stresses in the cellular, extracellular and blood vessel phases by $\sigma_{\alpha}, \sigma_{\beta}$, and $\sigma_{\gamma}$, and neglecting inertia ${ }^{\ddagger}$, we write

$$
\begin{aligned}
& \frac{\partial}{\partial x}\left(\alpha \sigma_{\alpha}\right)+F_{\alpha}=0, \\
& \frac{\partial}{\partial x}\left(\beta \sigma_{\beta}\right)+F_{\beta}=0, \\
& \frac{\partial}{\partial x}\left(\gamma \sigma_{\gamma}\right)+F_{\gamma}=0 .
\end{aligned}
$$

In (5)-(7), $F_{\alpha}, F_{\beta}$ and $F_{\gamma}$ denote the momentum sources and sinks for each phase.

As discussed in the introduction, we do not explicitly track the oxygen tension through the tumour mass; we assume instead that it is a known function of the local blood vessel volume fraction within the tumour.

\footnotetext{
${ }^{\dagger}$ Here we mean the actual density of each phase rather than the average density.

${ }^{\ddagger}$ We may check, a posteriori, that the Reynolds number for the flow, Re $=\rho L U / \mu_{\alpha} \sim 10^{-2}$.
} 
Thus, our model consists of (1)-(7), i.e. seven equations for the nine unknowns $\alpha, \beta, \gamma, u_{\alpha}, u_{\beta}$, $u_{\gamma}, \sigma_{\alpha}, \sigma_{\beta}$ and $\sigma_{\gamma}$. We therefore require two constitutive laws to determine the model fully. In the rest of this section, we will establish functional forms for $q_{\alpha}, q_{\beta}, q_{\gamma}, \sigma_{\alpha}, \sigma_{\beta}, \sigma_{\gamma}, F_{\alpha}, F_{\beta}$, and $F_{\gamma}$, and the initial and boundary conditions.

2.1. Constitutive laws. In order to describe the biophysical effects that occur within the tumour, we need to make choices for $q_{i}, \sigma_{i}$ and $F_{i}(i=\alpha, \beta, \gamma)$ which are physically realistic while minimising the mathematical complexity of the problem. When stating the constitutive laws for the stress tensors, we shall introduce a further three dependent variables: the tumour cell pressure $p_{\alpha}$, the blood vessel phase pressure $p_{\gamma}$, and the extracellular material pressure $p_{\beta}$, and therefore will also have to specify further relations describing these pressures (note that because of our one-dimensional geometry the stress tensors reduce to scalar functions and that the pressures are therefore equivalent to the stresses in each phase). Our aim is, by the end of this section, to have developed a closed system of equations.

Stress tensors. We identify with the stress in each phase an isotropic pressure so

$$
\sigma_{\alpha}=-p_{\alpha}, \quad \sigma_{\beta}=-p_{\beta}, \quad \sigma_{\gamma}=-p_{\gamma},
$$

where $p_{\alpha}, p_{\beta}$ and $p_{\gamma}$ are the average pressures in the three phases. Note that, with our model formulation, if we wished to modify the mechanical behaviour of any of the phases, for example, to assume that the tumour cell phase behaved like a viscous liquid, we would merely modify the stress relations given in (8), so that we would set, for example $\sigma_{\alpha}=-p_{\alpha}+\mu \frac{\partial u_{\alpha}}{\partial x}$. This extends to two dimensions in the obvious way, taking care to account for the fact that $\nabla \cdot \mathbf{u}_{\alpha} \neq 0$ because of tumour cell proliferation and death.

In order to close the model equations we specify two constitutive relationships. As in Breward et al. (2002), we assume that the cells behave as a fluid but, in addition, interact with each other. Thus, the first of these constitutive relationships reads

$$
p_{\alpha}=p_{\beta}+\Sigma_{\alpha}
$$

where $\Sigma_{\alpha}$ describes the pressure due to cell-cell interactions. We suppose that there is a certain volume fraction, $\alpha^{*}$ say, at which the cells are at their natural, close-packing density. At higher volume fractions, the cells' membranes deform and they experience stress. This pressure becomes infinite as the cell volume fraction approaches $\alpha=1$. Under such conditions (when $\alpha>\alpha^{*}$ ), the cells will attempt to move in order to reduce their stress. When $\alpha<\alpha^{*}$, we assume that the cells are sufficiently disperse that they experience no interactions. Thus, we choose the following functional form for $\Sigma_{\alpha}$ :

$$
\Sigma_{\alpha}=\left\{\begin{array}{ccc}
\lambda \frac{\left(\alpha-\alpha^{*}\right)}{(1-\alpha)^{2}} & \text { if } & \alpha \geq \alpha^{*}, \\
0 & \text { if } & \alpha<\alpha^{*} .
\end{array}\right.
$$

In (10), we call $\lambda$ the tension constant. The tension constant is a measure of the cells' affinity for their natural volume fraction $\alpha^{*}$. As $\lambda$ increases, the force driving the tumour cells to their natural volume fraction increases.

The second constitutive relationship concerns the average pressure in the blood vessel phase. Since our model is spatially averaged and does not concern details of the flow through individual blood vessels (along which the pressure might drop slightly), our consitutive law is simply

$$
p_{\gamma}=p_{\gamma}^{*},
$$


where $p_{\gamma}^{*}$ is the externally-set pressure. If we extended our model in to two dimensions and allowed for directionality of blood flow so that $p_{\gamma}=p_{\gamma}(\mathbf{x}, t)$, we would lose the obvious symmetry seen in the experiments. We note also that we do not consider details of vessel compliance since we believe these are important only on the vessel's microscale (see Breward et al. (2001))

Volume source and sink terms. Within a vascular tumour, volume may be introduced via the vasculature and removed via the lymph drainage system. Thus, in general, within the tumour the net volume growth rate $q=q_{\alpha}+q_{\beta}+q_{\gamma} \neq 0$, whereas in an avascular tumour $q=0$ since flow through the outer tumour boundary is the only mechanism by which its volume can increase or decrease (Breward et al., 2002).

We suppose that the volume fraction of tumour cells increases by proliferation, and decreases due to cell death (we do not distinguish between necrosis and apoptosis as separate death mechanisms). For proliferation to occur, we must have tumour cells already present, extracellular material present to provide the material for volumetric growth of the cells, and blood vessels in the vicinity to provide the necessary oxygen. While there are many nonlinear forms (as a function of $\alpha, \beta$ and $\gamma$ ) which exhibit these properties, we choose the simplest of these, assuming that the proliferation rate is proportional to the product of the three volume fractions. We assume that cell death is proportional to the number of cells present and that it depends on the blood vessel volume fraction. In areas that are well vascularised, we assume that there is a very small level of cellular death (by apoptosis), and that, as the blood vessel volume fraction falls there is a small increase in the death rate until the blood vessel volume fraction becomes 'very low'. When there are few viable vessels in a region, we assume that there is extensive tumour cell death (which can be attributed to oxygen-induced necrosis). Further the rate of cellular death is assumed maximal when $\gamma=0$. We therefore choose to write

$$
q_{\alpha}=k_{1} \alpha \beta \gamma-k_{2} \alpha\left(1-\frac{\gamma}{\gamma_{c}+\gamma}\right),
$$

where $k_{1}$ is the rate constant for cell proliferation, $k_{2}$ is the maximum rate at which cell death can occur and $\gamma_{c}$ is the volume fraction of blood vessels at which the death rate is half maximal.

We suppose that the blood vessel volume fraction increases due to angiogenesis and decreases due to vessel occlusion. We assume that angiogenesis requires the presence of both tumour cells and blood vessels and that the rate of angiogenesis is maximal at some small value of tumour cell volume fraction, $\alpha_{m}$ say. We further assume that vessels become occluded if the pressure exerted on them by the cells surrounding them, $\alpha p_{\alpha}$ exceeds a critical pressure, $p_{\text {crit }}$ say. Note that we would expect $p_{\text {crit }}$ to increase with vessel wall strength (which would be important if we were to consider co-opted and neo-vessels separately). We write

$$
q_{\gamma}=k_{4} \frac{\alpha \gamma}{\alpha_{m}^{2}+\alpha^{2}}-k_{3} \gamma \mathcal{G}\left(\alpha p_{c}-p_{c r i t}\right),
$$

where $k_{3}$ and $k_{4}$ are rate constants for occlusion and angiogenesis respectively. In (13), $\mathcal{G}$ is a smooth transition function (for example $\mathcal{G}(\theta)=(1+\tanh (\theta / \epsilon)) / 2$ for some $\epsilon \ll 1$ ) that switches vessel death on smoothly as $\alpha p_{c}$ approaches $p_{\text {crit }}$ from above. We note that the volume of matter needed for angiogenesis is assumed to be supplied from within the vasculature (i.e. there is no balancing sink term in the mass balance equations for $\alpha$ and $\beta$ ).

We suppose that the volume fraction of extracellular material decreases due to tumour cell birth, and increases due to tumour cell death. We assume that some of the material resulting from cell death is removed by the lymph drainage system (present in the $\beta$ phase), providing $\beta$ exceeds $\beta_{L}$ (i.e. the osmotic pressure is in the correct direction and the vessels are able to function - see below). Further, we assume that water can enter this phase from the blood vessels, providing $\beta$ is 
less than $\beta_{\gamma}$. We also include the increase in $\beta$ due to the death of blood vessels. Taking all these mechanisms into account, we write

$$
\begin{gathered}
q_{\beta}=k_{2} \alpha\left(1-\frac{\gamma}{\gamma_{c}+\gamma}\right)+k_{3} \gamma \mathcal{G}\left(\alpha p_{c}-p_{\text {crit }}\right)-k_{1} \alpha \beta \gamma+k_{5} \gamma\left(\beta_{B}-\beta\right) \mathcal{H}\left(\beta_{B}-\beta\right) \\
-k_{6}\left(\beta-\beta_{L}\right) \mathcal{H}\left(\beta-\beta_{L}\right) \mathcal{G}\left(p_{\text {crit }}-\alpha p_{c}\right),
\end{gathered}
$$

where $k_{5}$ and $k_{6}$ are osmotic rate constants, and $\mathcal{H}$ is a heaviside function $\left(\mathcal{H}\left(x-x_{0}\right)=1\right.$ if $x \geq x_{0}$, else it is zero).

On first inspection, it might appear to be inconsistent to include both $\mathcal{G}$ and $\mathcal{H}$ in our model. However, the processes described using these two functions are distinct. We use $\mathcal{H}$ in places where a process is either on or off ( $\mathrm{eg}$ to stop flow of liquid from the lymph drainage system back into the tumour mass), while we use $\mathcal{G}$ in places where there is a smooth transition. Finally, we note that, by including the $\mathcal{G}$ factor in (14), we have assumed that the lymph drainage vessels will collapse (and, hence, lymph drainage will cease) at the same pressure as the blood vessels become occluded.

Momentum source terms. We suppose that there are momentum sources due to the pressures and that differential movement of any two phases manifests itself as a Darcy-style drag term. Accordingly, we set

$$
\begin{aligned}
& F_{\alpha}=p_{\alpha} \frac{\partial \alpha}{\partial x}+d_{1} \alpha \beta\left(u_{\beta}-u_{\alpha}\right)+d_{2} \alpha \gamma\left(u_{\gamma}-u_{\alpha}\right), \\
& F_{\beta}=p_{\beta} \frac{\partial \beta}{\partial x}-d_{1} \alpha \beta\left(u_{\beta}-u_{\alpha}\right)+d_{3} \beta \gamma\left(u_{\gamma}-u_{\beta}\right), \\
& F_{\gamma}=p_{\gamma} \frac{\partial \gamma}{\partial x}-d_{2} \alpha \gamma\left(u_{\gamma}-u_{\alpha}\right)-d_{3} \beta \gamma\left(u_{\gamma}-u_{\beta}\right),
\end{aligned}
$$

In (15-17) $d_{1}, d_{2}$ and $d_{3}$ are drag coefficients associated with the ease with which the associated phases can move past each other. We have assumed that the drag terms are proportional to the product of the volume fractions of the two phases involved, (i.e. there is no drag if either species is not present). Other permeabilities may be more physically realistic (for example, we could use the Carman-Kozeny relationship presented in Fowler (1997)). However, for simplicity, we proceed with the forms for the drags stated above.

2.2. Initial and boundary conditions. We assume that the tumour's outer boundary moves at the same speed as the tumour cells on the boundary and so we fix

$$
l_{t}=\left.u_{\alpha}\right|_{x=l} .
$$

We then suppose that the width of the tumour and the initial volume fraction of the cells and blood vessels are known, so that

$$
l=L_{0}, \quad \alpha=\alpha_{0}(x) \quad \text { and } \quad \gamma=\gamma_{0}(x) \quad \text { at } \quad t=0 .
$$

Note that, with this information, we automatically know the initial distribution of the extracellular material $\beta(x, 0)$ using (4). We assume symmetry about the tumour centre, so that

$$
u_{\alpha}=u_{\beta}=u_{\gamma}=0 \quad \text { at } \quad x=0 .
$$


At the tumour boundary we must consider how the tumour interacts with the tissue in which it is growing. In this paper, we assume that the external tissue exerts no stress on the tumour. While this may be inappropriate for a tumour embedded deeply into a tissue it seems reasonable for a tumour implanted directly beneath the skin of a mouse. (It would be straightforward to incorporate external stresses into our model in order to describe situations in which the tumour was located deeper within the host tissue, see Chen et al. (2001), for example.) We assume that water may pass freely across the tumour periphery and, thus, the pressure in the extracellular material may be set equal to zero there (i.e. our pressure is set so that $P_{\text {ext }}=0$ ). Then, balancing stresses at the free surface, equations (9) and (10) imply that the volume fraction of the tumour cells must be less than or equal to the natural volume fraction $\alpha^{*}$.

We assume continuity of blood vessel density across the tumour boundary, and we suppose that the volume fraction of blood vessels outside the tumour is constant, with value $\gamma^{*}$ say. Finally, we suppose that this $\gamma^{*}$ is sufficient to sustain tumour cell proliferation when the volume fraction of tumour cells is at $\alpha^{*}$. In this case, there is an initial transient during which the volume fraction at the tumour's edge increases from $\alpha_{0}\left(L_{0}\right)$ to $\alpha^{*}$, after which $\alpha=\alpha^{*}$. We shall not concern ourselves with this initial transient in this paper, rather we shall set $\alpha_{0}\left(L_{0}\right)=\alpha^{*}$, and we therefore write

$$
p_{\beta}=0, \quad \alpha=\alpha^{*} \quad \text { and } \quad \gamma=\gamma^{*} \quad \text { at } \quad x=l .
$$

In summary, our model consists of the conservation of mass equations (1-3), with sources given by (12-13), the no-voids condition (4), the conservation of momentum equations (5-7), with stresses defined in (8) and sources defined in (15-16), the constitutive relationships given in $(9,11)$, the equation for the evolution of the tumour boundary (18) and the initial and boundary conditions given in (19-21). We note that the model comprises ten equations for the ten unknowns $\alpha, \beta, \gamma$, $u_{\alpha}, u_{\beta}, u_{\gamma}, p_{\alpha}, p_{\beta}, p_{\gamma}$ and $l$. In the next section we show how the model may be reduced to two equations for $\alpha$ and $\gamma$.

\section{Model simplification}

The model developed in $\S 2$ comprises ten equations for ten unknowns and is thus unwieldy. We show how the model can be reformulated in terms of only three of the dependent variables; we choose to retain the volume fraction of tumour cells, $\alpha$, the volume fraction of blood vessels, $\gamma$, and the average volume flux of material, $u_{m i x}$ where

$$
u_{m i x}=\alpha u_{\alpha}+\beta u_{\beta}+\gamma u_{\gamma}
$$

Note that we have chosen without loss of generality, not to track $\beta$ explicitly: we may calculate it a posteriori using (4).

First we add (1-3), to obtain

$$
\frac{\partial}{\partial x}\left(u_{\text {mix }}\right)=q_{\alpha}+q_{\beta}+q_{\gamma}=q,
$$

which we may integrate, subject to (20), to obtain

$$
u_{m i x}=\int_{0}^{x} q d x .
$$


Similarly, adding (5-7), and substituting for the stresses in each phase using (8), we find that

$$
(\alpha+\beta) \frac{\partial p_{\beta}}{\partial x}=-\alpha \frac{\partial \Sigma_{\alpha}}{\partial x},
$$

i.e. $p_{\beta}$ is a function of the volume fractions. Note that we keep $\beta=1-\alpha-\gamma$ in this equation, and those to follow, for convenience. We use (7), (11) and (17) to obtain the following relationship between the velocities, namely

$$
u_{\gamma}=\frac{d_{2} \alpha u_{\alpha}+d_{3} \beta u_{\beta}}{d_{2} \alpha+d_{3} \beta} .
$$

Rearranging (6) and substituting for $\sigma_{\beta}$ using (8), we find that either $\beta=0$ (which we reject since regions in which $\beta=0$ can only be transient: where $\beta=0$ there is no tumour cell proliferation but tumour cell death still occurs, creating extracellular material so that $\beta>0$ ), or

$$
\left(d_{1} \alpha+d_{3} \gamma\right) u_{\beta}-d_{1} \alpha u_{\alpha}-d_{3} \gamma u_{\gamma}=-\frac{\partial p_{\beta}}{\partial x}=\frac{\alpha}{\alpha+\beta} \frac{\partial}{\partial x} \Sigma_{\alpha},
$$

Equations (22), (26) and (27) are three simultaneous equations for $u_{\alpha}, u_{\gamma}$ and $u_{\beta}$. Solving for $u_{\alpha}$ and $u_{\beta}$, we find that

$$
u_{\alpha}=u_{m i x}-\frac{\beta\left(d_{3}+\left(d_{2}-d_{3}\right) \alpha\right)}{(\alpha+\beta)\left(d_{1} d_{3} \beta+d_{1} d_{2} \alpha+d_{2} d_{3} \gamma\right)} \frac{\partial \Sigma_{\alpha}}{\partial x},
$$

and

$$
u_{\beta}=u_{m i x}+\frac{\alpha\left(d_{2}+\left(d_{3}-d_{2}\right) \beta\right)}{(\alpha+\beta)\left(d_{1} d_{3} \beta+d_{1} d_{2} \alpha+d_{2} d_{3} \gamma\right)} \frac{\partial \Sigma_{\alpha}}{\partial x} .
$$

Subsituting for $u_{\alpha}$ and $u_{\beta}$ into (1) and (3), we have

$$
\frac{\partial \alpha}{\partial t}+\frac{\partial}{\partial x}\left(\alpha u_{m i x}\right)-\frac{\partial}{\partial x}\left(\frac{\alpha(1-\alpha-\gamma)\left(d_{3}+\left(d_{2}-d_{3}\right) \alpha\right)}{(1-\gamma)\left(d_{1} d_{3}(1-\alpha-\gamma)+d_{1} d_{2} \alpha+d_{2} d_{3} \gamma\right)} \frac{\partial \Sigma_{\alpha}}{\partial x}\right)=q_{\alpha}
$$

and

$$
\frac{\partial \gamma}{\partial t}+\frac{\partial}{\partial x}\left(\gamma u_{m i x}\right)+\frac{\partial}{\partial x}\left(\frac{\alpha(1-\alpha-\gamma)\left(d_{3}-d_{2}\right)}{(1-\gamma)\left(d_{1} d_{3}(1-\alpha-\gamma)+d_{1} d_{2} \alpha+d_{2} d_{3} \gamma\right)} \frac{\partial \Sigma_{\alpha}}{\partial x}\right)=q_{\gamma}
$$

and the initial and boundary conditions are given by

$$
\begin{aligned}
& l=L_{0} \quad \alpha=\alpha_{0}(x) \quad \text { and } \quad \gamma=\gamma_{0}(x) \quad \text { at } \quad t=0 \text {, } \\
& p_{\beta}=0, \quad \alpha=\alpha^{*} \quad \text { and } \quad \gamma=\gamma^{*} \quad \text { at } \quad x=1 \text {, } \\
& \frac{d l}{d t}=\left.u_{\alpha}\right|_{x=l},
\end{aligned}
$$

with (20) giving

$$
\frac{\partial \Sigma_{\alpha}}{\partial x}=0 \quad \text { at } \quad x=0 .
$$

We remark that the behaviour of the cells within the tumour are influenced by non-local effects in two different ways. Firstly, the tumour cells can be advected with the mixture velocity $u_{\text {mix }}$. Secondly, the cell-cell interactions manifest themselves as a nonlinear diffusion term in (30), which will transport cells down gradients in cellular density. We note also that other authors (for example Gatenby and Gawlinski (1996) and Sherratt (2000)) use nonlinear diffusion equations as the starting point for their modelling; by contrast we start by modelling the stresses in the tumour constituents and arrive at a situtation in which cells move by a diffusion-like process. 
3.1. Nondimensionalisation. Remembering that $\alpha$ and $\gamma$ are volume fractions and therefore dimensionless quantities, we scale equations (30)-(35) by introducing the dimensionless variables $t^{\prime}, x^{\prime}, l^{\prime}, u_{m i x}^{\prime}, p_{\alpha}^{\prime}$ and $p_{\beta}^{\prime}$ where

$$
\begin{gathered}
t=\frac{1}{\alpha^{*} \gamma^{*} k_{1}\left(1-\alpha^{*}-\gamma^{*}\right)} t^{\prime}, \quad x=L_{0} x^{\prime}, \quad l=L_{0} l^{\prime}, \\
u_{m i x}=L_{0} \alpha^{*} \gamma^{*} k_{1}\left(1-\alpha^{*}-\gamma^{*}\right) u_{m i x}^{\prime}, \quad\left(p_{\alpha}, p_{\beta}\right)=\lambda\left(p_{\alpha}^{\prime}, p_{\beta}^{\prime}\right) .
\end{gathered}
$$

Dropping primes, the resulting problem reads

$$
\begin{gathered}
\frac{\partial \alpha}{\partial t}+\frac{\partial}{\partial x}\left(\alpha u_{m i x}\right)-D \frac{\partial}{\partial x}\left[\left(d_{3}^{*}+\left(1-d_{3}^{*}\right) \alpha\right) F(\alpha, \gamma)\right]=q_{\alpha}^{*} \\
\frac{\partial \gamma}{\partial t}+\frac{\partial}{\partial x}\left(\gamma u_{\text {mix }}\right)+D\left(1-d_{3}^{*}\right) \frac{\partial}{\partial x}[F(\alpha, \gamma)]=q_{\gamma}^{*} \\
\frac{\partial u_{\text {mix }}}{\partial x}=q_{\alpha}^{*}+q_{\beta}^{*}+q_{\gamma}^{*}=q^{*}
\end{gathered}
$$

where

$$
\begin{gathered}
F(\alpha, \gamma)=\frac{\alpha(1-\alpha-\gamma)}{(1-\gamma)\left(\alpha+d_{3}^{*}(1-\alpha-\gamma)+\frac{d_{3}^{*}}{d_{1}^{*} \gamma}\right)} \frac{\partial}{\partial x}\left(\frac{\left(\alpha-\alpha^{*}\right) \mathcal{H}\left(\alpha-\alpha^{*}\right)}{(1-\alpha)^{2}}\right), \\
q_{\alpha}^{*}=\frac{\alpha \gamma(1-\alpha-\gamma)}{\alpha^{*} \gamma^{*}\left(1-\alpha^{*}-\gamma^{*}\right)}-k_{2}^{*} \alpha\left(1-\frac{\gamma}{\gamma_{c}+\gamma}\right), \\
q_{\beta}^{*}=k_{2}^{*} \alpha\left(1-\frac{\gamma}{\gamma_{c}+\gamma}\right)+k_{3}^{*} \gamma \mathcal{G}\left(\alpha p_{c}-p_{c r i t}\right)-\frac{\alpha \gamma(1-\alpha-\gamma)}{\alpha^{*} \gamma^{*}\left(1-\alpha^{*}-\gamma^{*}\right)} \\
+k_{5}^{*} \gamma\left(\beta_{B}-(1-\alpha-\gamma)\right) \mathcal{H}\left(\beta_{B}-(1-\alpha-\gamma)\right)-k_{6}^{*}\left((1-\alpha-\gamma)-\beta_{L}\right) \mathcal{H}\left((1-\alpha-\gamma)-\beta_{L}\right) \mathcal{G}\left(p_{c r i t}-\alpha p_{c}\right), \\
q_{\gamma}^{*}=k_{4}^{*} \frac{\alpha \gamma}{\alpha_{m}^{2}+\alpha^{2}}-k_{3}^{*} \gamma \mathcal{G}\left(\alpha p_{c}-p_{c r i t}\right),
\end{gathered}
$$

and, therefore,

$$
\begin{gathered}
q^{*}=k_{4}^{*} \frac{\alpha \gamma}{\alpha_{m}^{2}+\alpha^{2}}+k_{5}^{*} \gamma\left(\beta_{B}-(1-\alpha-\gamma)\right) \mathcal{H}\left(\beta_{B}-(1-\alpha-\gamma)\right) \\
-k_{6}^{*}\left((1-\alpha-\gamma)-\beta_{L}\right) \mathcal{H}\left((1-\alpha-\gamma)-\beta_{L}\right) \mathcal{G}\left(p_{c r i t}-\alpha p_{c}\right) .
\end{gathered}
$$

We have introduced eight nondimensional parameters in (38-44), defined as

$$
\begin{gathered}
D=\frac{\lambda}{d_{1} k_{1} L_{0}^{2} \alpha^{*} \gamma^{*}\left(1-\alpha^{*}-\gamma^{*}\right)}, \quad d_{1}^{*}=\frac{d_{1}}{d_{2}}, \quad d_{3}^{*}=\frac{d_{3}}{d_{2}}, \\
k_{2}^{*}=\frac{k_{2}}{k_{1} \alpha^{*} \gamma^{*}\left(1-\alpha^{*}-\gamma^{*}\right)}, \quad k_{3}^{*}=\frac{k_{3}}{k_{1} \alpha^{*} \gamma^{*}\left(1-\alpha^{*}-\gamma^{*}\right)}, \quad k_{4}^{*}=\frac{k_{4}}{k_{1} \alpha^{*} \gamma^{*}\left(1-\alpha^{*}-\gamma^{*}\right)}, \\
k_{5}^{*}=\frac{k_{5}}{k_{1} \alpha^{*} \gamma^{*}\left(1-\alpha^{*}-\gamma^{*}\right)}, \quad k_{6}^{*}=\frac{k_{6}}{k_{1} \alpha^{*} \gamma^{*}\left(1-\alpha^{*}-\gamma^{*}\right)} .
\end{gathered}
$$


We note that the tumour width increases via

$$
\frac{d l}{d t}=u_{m i x}-\frac{D}{\alpha}\left(d_{3}^{*}+\left(1-d_{3}^{*}\right) \alpha\right) F(\alpha, \gamma) \quad \text { at } \quad x=l,
$$

and that the pressures satisfy

$$
\frac{\partial p_{\beta}}{\partial x}=-\frac{\alpha}{1-\gamma} \frac{\partial}{\partial x}\left(\frac{\alpha-\alpha^{*}}{(1-\alpha)^{2}} \mathcal{H}\left(\alpha-\alpha^{*}\right)\right), \quad p_{\alpha}=p_{\beta}+\frac{\alpha-\alpha^{*}}{(1-\alpha)^{2}} \mathcal{H}\left(\alpha-\alpha^{*}\right) .
$$

The initial and boundary conditions read

$$
\begin{aligned}
& l=1, \quad \alpha=\alpha_{0}, \quad \gamma=\gamma_{0} \quad \text { at } \quad t=0, \\
& p_{\beta}=0, \quad u_{\text {mix }}=\frac{\partial \Sigma_{\alpha}}{\partial x}=0 \quad \text { at } \quad x=0 \text {, } \\
& \alpha=\alpha^{*}, \quad \gamma=\gamma^{*} \quad \text { at } \quad x=l \text {. }
\end{aligned}
$$

3.2. Transformation of problem onto fixed domain. Before embarking on numerical simulations of the governing equations it is useful to recast the problem from the moving domain $0 \leq x \leq l(t)$ onto $0 \leq \xi \leq 1$. We set $\tau=t, \xi=x / l(t)$, and then the resulting problem reads

$$
\begin{gathered}
\frac{\partial \alpha}{\partial \tau}+\frac{1}{l}\left(u_{m i x}-\frac{d l}{d \tau} \xi\right) \frac{\partial \alpha}{\partial \xi}-\frac{D}{l^{2}} \frac{\partial}{\partial \xi}\left(\left(d_{3}^{*}+\left(1-d_{3}^{*}\right) \alpha\right) \mathcal{F}(\alpha, \gamma)\right)=q_{\alpha}^{*}-\alpha q^{*} \\
\frac{\partial \gamma}{\partial \tau}+\frac{1}{l}\left(u_{m i x}-\frac{d l}{d \tau} \xi\right) \frac{\partial \gamma}{\partial \xi}+\frac{D}{l^{2}}\left(1-d_{3}^{*}\right) \frac{\partial \mathcal{F}(\alpha, \gamma)}{\partial \xi}=q_{\gamma}^{*}-\gamma q^{*} \\
\frac{\partial u_{m i x}}{\partial \xi}=l q^{*}
\end{gathered}
$$

and

$$
\frac{d l}{d \tau}=u_{m i x}-\frac{D}{\alpha}\left(d_{3}^{*}+\left(1-d_{3}^{*}\right) \alpha\right) \hat{F} \quad \text { at } \quad \xi=1,
$$

where

$$
\mathcal{F}(\alpha, \gamma)=\frac{\alpha(1-\alpha-\gamma)}{(1-\gamma)\left(\alpha+d_{3}^{*}(1-\alpha-\gamma)+\frac{d_{3}^{*}}{d_{1}^{*}} \gamma\right)} \frac{\partial}{\partial \xi}\left(\frac{\left(\alpha-\alpha^{*}\right) \mathcal{H}\left(\alpha-\alpha^{*}\right)}{(1-\alpha)^{2}}\right) .
$$

The initial and boundary conditions become

$$
\begin{array}{r}
l=1, \alpha=\alpha_{0}, \gamma=\gamma_{0} \text { at } \tau=0, \\
u_{\text {mix }}=\frac{\partial \Sigma_{\alpha}}{\partial \xi}=0 \text { at } \xi=0, \\
\alpha=\alpha^{*}, \gamma=\gamma^{*} \text { at } \xi=1 .
\end{array}
$$




\section{Possible growth regimes}

In this section we consider several growth regimes for which the model equations presented in (52-59) simplify. First we set all the drag coefficients equal, so that $d_{1}^{*}=d_{3}^{*}=1$. We find that the phase speeds reduce to

$$
u_{\alpha}=u_{m i x}-\frac{D(1-\alpha-\gamma)}{l(1-\gamma)} \frac{\partial \Sigma_{\alpha}}{\partial \xi}, \quad u_{\beta}=u_{m i x}+\frac{D \alpha}{l(1-\gamma)} \frac{\partial \Sigma_{\alpha}}{\partial \xi}, \quad u_{\gamma}=u_{m i x}
$$

Thus, in this limit, the blood vessels are advected simply with the mixture velocity: they do not move as a result of the stress induced in the tumour. We find the resulting system of equations reads

$$
\begin{gathered}
\frac{\partial \alpha}{\partial \tau}+\frac{1}{l}\left(u_{m i x}-\frac{d l}{d \tau} \xi\right) \frac{\partial \alpha}{\partial \xi}=\frac{D}{l^{2}} \frac{\partial}{\partial \xi}\left(\frac{\alpha(1-\alpha-\gamma)}{1-\gamma} \frac{\partial \Sigma_{\alpha}}{\partial \xi}\right)+q_{\alpha}^{*}-\alpha q^{*} \\
\frac{\partial \gamma}{\partial \tau}+\frac{1}{l}\left(u_{m i x}-\frac{d l}{d \tau} \xi\right) \frac{\partial \gamma}{\partial \xi}=q_{\gamma}^{*}-\gamma q^{*}
\end{gathered}
$$

where

$$
\frac{\partial u_{\operatorname{mix}}}{\partial \xi}=l q^{*}
$$

and

$$
\begin{array}{r}
\alpha=\alpha_{0}(\xi), \quad \gamma=\gamma_{0}(\xi), \text { at } \tau=0, \\
u_{\text {mix }}=0 \text { at } \xi=0, \\
\alpha=\alpha^{*}, \quad \gamma=\gamma^{*}, \text { at } \xi=1, \\
\frac{d l}{d \tau}=u_{\text {mix }}-\frac{D\left(1-\alpha^{*}-\gamma^{*}\right)}{l\left(1-\gamma^{*}\right)} \frac{\partial}{\partial \xi}\left(\frac{\left(\alpha-\alpha^{*}\right)}{(1-\alpha)^{2}}\right) \text { at } \xi=1 .
\end{array}
$$

Note that if we assume that the major contribution to the drag forces comes from cell-vessel interactions, so that $d_{1}^{*} \sim d_{3}^{*} \ll 1$, then the nonlinear diffusion coefficient in (61) and the convection term in (62) are modified. We do not consider this case further in this paper.

In the absence of a meaningful set of data, it is difficult to judge which areas of parameter space are most physically realistic. In the rest of this section we discuss various parameter limits of the above model for which the model equations simplify. In $\S 4.1$, we consider the case where $D \ll 1$. Initially, this case seems to yield a much simpler and analytically tractable problem. However, as we shall see, the resulting boundary layer problem that arises close to the free surface is as difficult to solve as the original model equations when $D \sim O(1)$. We include the analysis to illustrate that, in this case, the central region of the tumour exhibits spatially uniform volume fractions of cells and vessels, and that the tumour width increases solely due to the internal sources and sinks of mass. In the remaining subsections we increase the complexity of the model by introducing additional physical mechanisms in turn. In $\S 4.2$, we investigate the case where there are no internal sources or sinks of mass, and no vessel death. We show how the tumour vessel volume fraction can be determined explicitly, and derive a relationship, in terms of $\alpha^{*}$ and $\gamma_{c}$, which determines whether the tumour will grow. In $\S 4.3$, we allow material to enter the tumour through the blood vessels and in $\S 4.4$, we investigate the effects of vessel collapse on tumour structure. 
4.1. The limit as $D \rightarrow 0$. Referring to (46), $D \rightarrow 0$ when either the cells do not have an affinity for the natural density (i.e., $\lambda \rightarrow 0$ in terms of dimensional parameters) or the drag between each phase is very large $\left(\right.$ i.e. $\left.d_{1} \rightarrow \infty\right)$. In this limit we lose the diffusion-like term from the leading-order problem, which reads

$$
\begin{gathered}
\frac{\partial \alpha}{\partial \tau}+\frac{1}{l}\left(u_{m i x}-\frac{d l}{d \tau} \xi\right) \frac{\partial \alpha}{\partial \xi}=q_{\alpha}^{*}-\alpha q^{*} \\
\frac{\partial \gamma}{\partial \tau}+\frac{1}{l}\left(u_{m i x}-\frac{d l}{d \tau} \xi\right) \frac{\partial \gamma}{\partial \xi}=q_{\gamma}^{*}-\gamma q^{*} \\
\frac{\partial u_{m i x}}{\partial \xi}=l q^{*}
\end{gathered}
$$

with initial and boundary conditions

$$
\begin{aligned}
\alpha=\alpha_{0}(\xi) \quad & \text { at } & t=0, \\
u_{m i x}=0 & \text { at } & \xi=0, \\
\alpha=\alpha^{*}, \gamma=\gamma^{*} & \text { at } & \xi=1, \\
\frac{d l}{d \tau}=u_{\text {mix }} & \text { at } & \xi=1 .
\end{aligned}
$$

Since (74) holds, (68) and (69) provide ordinary differential equations for the evolution of $\alpha$ and $\gamma$ respecively at $\xi=1$.

Thus we are unable to specify boundary conditions (73) at $\xi=1$. We therefore have a boundary layer at the growing edge of the tumour. To analyse growth in the region close to the periphery, we make the scaling $\xi=1-\sqrt{D} \eta$. Employing this coordinate transform and substituting power series expansions for the dependent variables (of the form $\alpha=\alpha^{(0)}+\sqrt{D} \alpha^{(1)}$ ), we find, from (63), that

$$
u_{\text {mix }}^{(0)}(\eta)=u_{\text {mix }}^{\text {out }}(1)
$$

where the superscript ${ }^{\text {out }}$ denotes the solution to the outer problem described above. Taking care to include the $O(\sqrt{D})$ terms where appropriate, we find that the leading-order inner variables $\alpha^{(0)}$ and $\gamma^{(0)}$ satisfy

$$
\begin{gathered}
\frac{\partial \alpha^{(0)}}{\partial \tau}-\frac{1}{l^{(0)}}\left(\eta+\left.\frac{1-\alpha^{*}-\gamma^{*}}{l^{(0)}\left(1-\gamma^{*}\right)}{\frac{\partial \Sigma_{\alpha}}{\partial \eta}}^{(0)}\right|_{\eta=0}\right) \frac{\partial \alpha}{\partial \eta}^{(0)}-\frac{1}{l^{(0)}} \frac{\partial}{\partial \eta}\left(\frac{\alpha^{(0)}\left(1-\alpha^{(0)}-\gamma^{(0)}\right)}{1-\gamma^{(0)}} \frac{\partial \Sigma_{\alpha}}{\partial \eta}{ }^{(0)}\right)= \\
q_{\alpha}^{*(0)}-\alpha^{(0)} q^{*(0)} \\
\frac{\partial \gamma^{(0)}}{\partial \tau}-\frac{1}{l^{(0)}}\left(\eta+\left.\frac{1-\alpha^{*}-\gamma^{*}}{l^{(0)}\left(1-\gamma^{*}\right)} \frac{\partial \Sigma_{\alpha}{ }^{(0)}}{\partial \eta}\right|_{\eta=0}\right) \frac{\partial \gamma^{(0)}}{\partial \eta}=q_{\gamma}^{*(0)}-\gamma^{(0)} q^{*(0)} \\
\alpha^{(0)}=\alpha_{0}, \gamma^{(0)}=\gamma_{0}, \text { at } \tau=0 \\
\alpha^{(0)}=\alpha^{*}, \gamma^{(0)}=\gamma^{*} \text {, at } \eta=0 \\
\alpha^{(0)} \rightarrow \alpha^{\text {out }} \text { as } \eta \rightarrow \infty
\end{gathered}
$$


We may make some progress with the outer problem before having to consider the boundary layer. In fact, if the initial distributions $\alpha(\xi, 0)$ and $\gamma(\xi, 0)$ are spatially uniform, then $\alpha$ and $\gamma$ will remain spatially uniform for $\tau>0$. Thus $q=q^{*}(\tau)$ and

$$
u_{m i x}=l q^{*} \xi
$$

Therefore the width of the tumour increases as

$$
l=e^{\int_{0}^{\tau} q^{*}\left(\tau^{\prime}\right) d \tau^{\prime}}
$$

and the evolution of $\alpha$ and $\gamma$ is given by

$$
\frac{\partial \alpha}{\partial \tau}=q_{\alpha}^{*}-q^{*}, \quad \frac{\partial \gamma}{\partial \tau}=q_{\gamma}^{*}-\gamma q^{*} .
$$

These equations may be solved numerically relatively easily, once the pressure field has been determined. With $\alpha$ constant, the solution to the pressure equations in this region read

$$
p_{\beta}=p_{\beta}^{*}(\tau), \quad p_{\alpha}=p_{\beta}^{*}(\tau)+\Sigma_{\alpha}(\alpha),
$$

where $p_{\beta}^{*}(\tau)$ must be found by solving the inner problem and calculating the pressure as $\eta \rightarrow \infty$. The inner problem given by (76-80) appears to be as difficult to solve as the $D \sim O(1)$ problem. Thus we do not attempt to solve it in this paper. Rather, we shall solve the full problem for small $D$ in $\S 4.2$ and see that it manifests the characteristics of the solution described here.

4.2. Tumour growth when there are no internal sources of mass and no vessel death. In this section we investigate how the tumour volume evolves when there is no birth or death of blood vessels and no flow of liquid in through the blood vessels or out through the lymph system (i.e., $k_{i}^{*}=0, i=3,4,5,6$ in equations (61)-(67)). In this case, we may determine the volume fraction of blood vessels explicitly. It is easy to see (from (62)) that $\gamma$ remains constant (and equals $\gamma_{k}$ say) along the characteristic curves, given by $\xi=\xi_{0} / l(\tau)$, for $0 \leq \xi_{0} \leq 1$. Thus, in terms of the original (nondimensionalised) physical variables, the initial conditions are propagated through time in the region $0 \leq x \leq 1$ while the boundary condition at $x=l$ influences the region $1 \leq x \leq l(t)$.

Using the fact that $\gamma_{k}=\gamma_{0}(\xi l(\tau))$ for $0 \leq \xi<1 / l, \gamma_{k}=\gamma^{*}$ for $1 / l(\tau) \leq \xi \leq 1$, our model reduces to a nonlinear parabolic equation for $\alpha$, namely

$$
\frac{\partial \alpha}{\partial \tau}-\frac{\xi}{l} \frac{d l}{d \tau} \frac{\partial \alpha}{\partial \xi}=\frac{D}{l^{2}} \frac{\partial}{\partial \xi}\left(\frac{\alpha\left(1-\gamma_{k}-\alpha\right)}{1-\gamma_{k}} \frac{\partial \Sigma_{\alpha}}{\partial \xi}\right)_{\xi}+\frac{\alpha \gamma_{k}\left(1-\gamma_{k}-\alpha\right)}{\alpha^{*} \gamma^{*}\left(1-\alpha^{*}-\gamma^{*}\right)}-k_{2}^{*} \alpha\left(1-\frac{\gamma_{k}}{\gamma_{c}+\gamma_{k}}\right)
$$

Thus we recover tumour growth similar to the oxygen independent growth described in Breward et al. (2002). Setting $\gamma_{0}=\gamma^{*}$, the solution is $\gamma_{k}=\gamma^{*}$. We pick $\gamma_{0}=\gamma^{*}(=0.2)$ so that the volume fraction of blood vessels remains at $\gamma^{*}, \forall t$, and we show how the volume fraction of tumour cells across the width of the tumour changes with time in figure 1 and how the width changes in figure 2. We see that the tumour cell volume fraction increases in the centre of the tumour, at the expense of extracellular material. The tumour cell volume fraction decreases to the natural volume fraction at the edge of the tumour. The stress induced by having cells above their natural volume fraction causes the tumour to grow, at an approximately linear rate. Note that, in the absence of angiogenesis, if we fix $\gamma_{0}=0$, we find that the tumour does not grow. Moreover, all the internal tumour cells die off quickly (since there are no vessels present to supply them with oxygen), leaving 


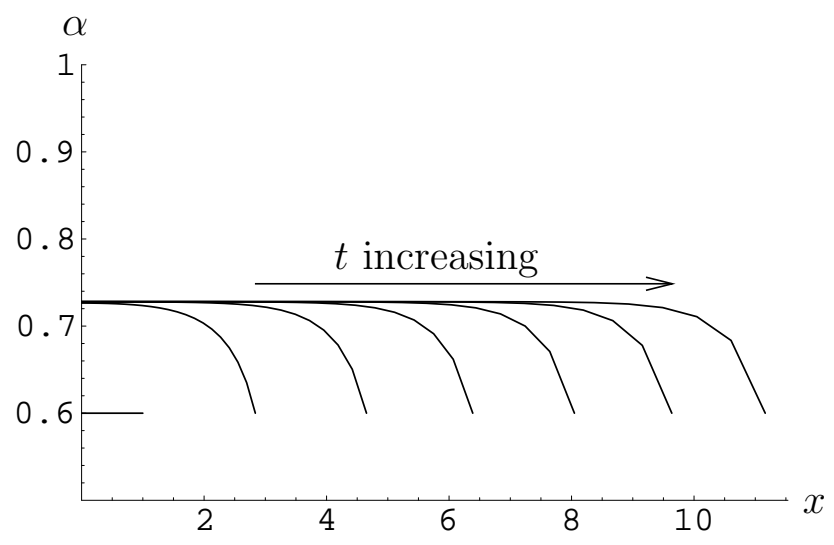

Figure 1. Graph showing how the volume fraction of tumour cells varies with distance from the centre of the tumour, for $t=0,4,8, \ldots \ldots, 24$. Here $k_{2}^{*}=3, D=1$ and all other parameters are set to zero.

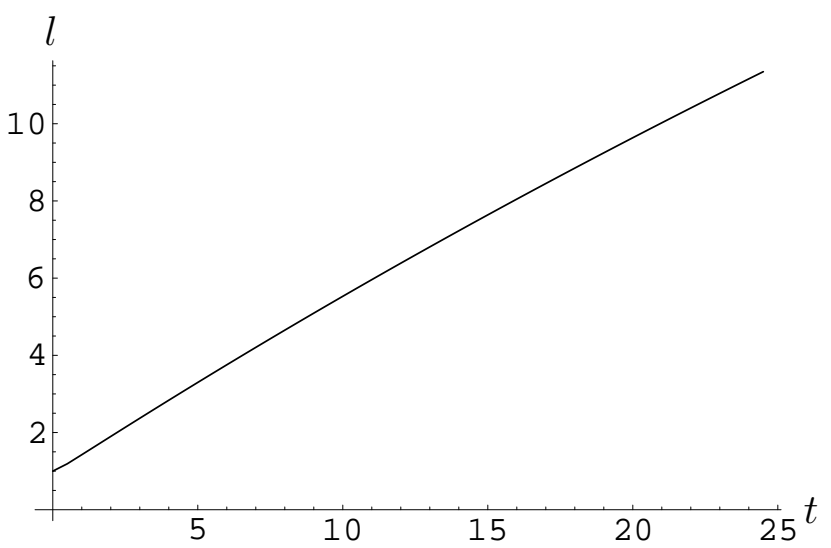

Figure 2. Graph showing how the width of the tumour changes with time. 


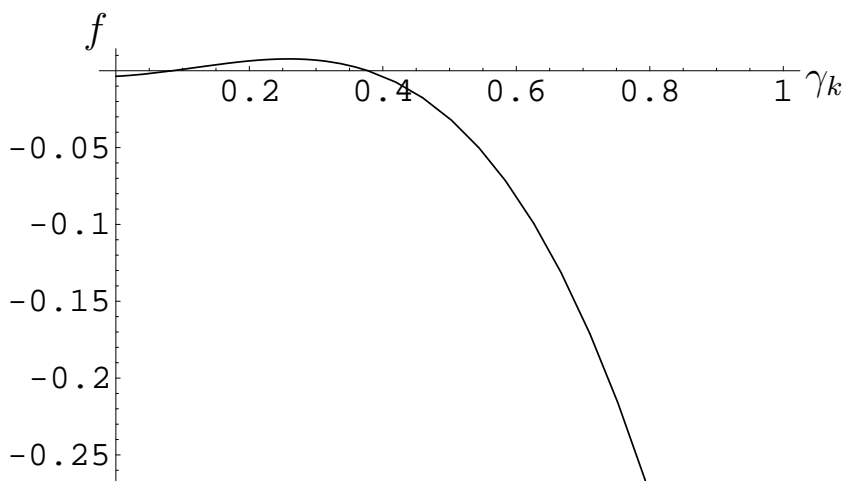

Figure 3. Graph showing how $f\left(\alpha^{*}, \gamma_{k}\right)$ varies with $\gamma_{k}$, for $\alpha^{*}$ fixed at 0.6 , and with $k_{2}^{\prime}=0.072$ and $\gamma_{c}=0.05$.

a rim of cells at the edge in contact with the external blood vessels. These cells remain at the natural volume fraction.

Suppose now we seek a solution in which the volume fraction of tumour cells remains constant, at the unstressed state $\alpha^{*}$. We use (85) to derive a relationship between $\alpha^{*}$ and $\gamma_{k}$ that must be satisfied to maintain this situation:

$$
\frac{\gamma_{k}\left(1-\gamma_{k}-\alpha^{*}\right)}{\gamma^{*}\left(1-\alpha^{*}-\gamma^{*}\right)}-k_{2}^{*}\left(\alpha^{*}, \gamma^{*}\right) \alpha^{*}\left(1-\frac{\gamma_{k}}{\gamma_{c}+\gamma_{k}}\right)=0
$$

where we emphasise that, as defined in (46), $k_{2}^{*}=k_{2}^{*}\left(\alpha^{*}, \gamma^{*}\right)$. Setting $k_{2}^{\prime}=k_{2} / k_{1}$, we find that the blood vessel volume fraction required to support $\alpha=\alpha^{*}$ satisfies

$$
f\left(\alpha^{*}, \gamma_{k}\right)=-\gamma_{k}^{3}+\left(1-\alpha^{*}-\gamma_{c}\right) \gamma_{k}^{2}+\gamma_{k}\left(1-\alpha^{*}\right) \gamma_{c}-k_{2}^{\prime} \gamma_{c}=0 .
$$

Equation (87) has one negative root which we dismiss as unphysical. The nature of the other roots depends on the parameters we choose. With $\alpha^{*}=0.6, k_{2}^{\prime}=0.072$ and $\gamma_{c}=0.05$, there are two physically relevant solutions; $\gamma_{k}=0.085$ and 0.38 (see figure 3). For $\gamma_{k}<0.085$, there are insufficient blood vessels present to support the growth of the tumour, whereas for $\gamma_{k}>0.38$ there is insufficient extracellular material (water) to support tumour growth. For intermediate values of $\gamma_{k}$ our tumour will grow.

We now determine (still assuming that $\alpha=\alpha^{*}, \gamma=\gamma_{k}$ throughout the tumour), for a given value of $\alpha^{*}$, the value of $\gamma_{k}$ which maximises the tumour's growth rate. Using (87) we deduce that the net tumour cell growth term is maximised when

$$
\gamma_{k}=\gamma_{\max }\left(\alpha^{*}\right)=\frac{\left(1-\alpha^{*}-\gamma_{c}\right)+\sqrt{1-2 \alpha^{*}+\alpha^{* 2}+\gamma_{c}\left(1-\alpha^{*}\right)+\gamma_{c}^{2}}}{3}
$$

We are able to verify that a maximum is realised when $\gamma_{k}=\gamma_{\max }\left(\alpha^{*}\right)$ by simply checking that

$$
f\left(\alpha^{*}, \gamma_{\max }\left(\alpha^{*}\right)\right) \geq 0
$$

We show how $f\left(\alpha^{*}, \gamma_{\max }\left(\alpha^{*}\right)\right)$ varies with $\alpha^{*}$ for $k_{2}^{\prime}=0.072$ and $\gamma_{c}=0.05$ in figure 4 . We see that there is a critical $\alpha^{*}(\sim 0.74$ in this case $)$ above which there is no physically realistic volume fraction of blood vessels which supports tumour growth in which the volume fraction of tumour cells 


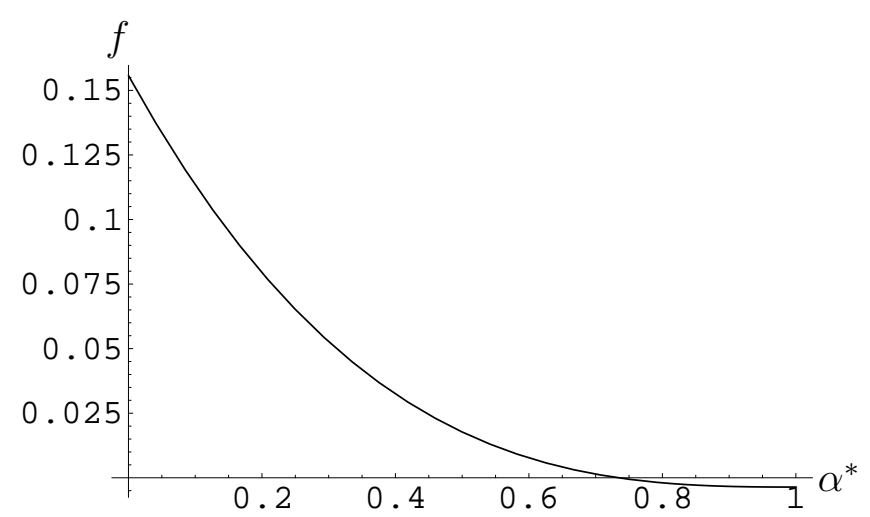

Figure 4. Graph showing how $f\left(\alpha^{*}, \gamma_{\max }\left(\alpha^{*}\right)\right)$ varies with $\alpha^{*}$, when $k_{2}^{\prime}=0.072$ and $\gamma_{c}=0.05$

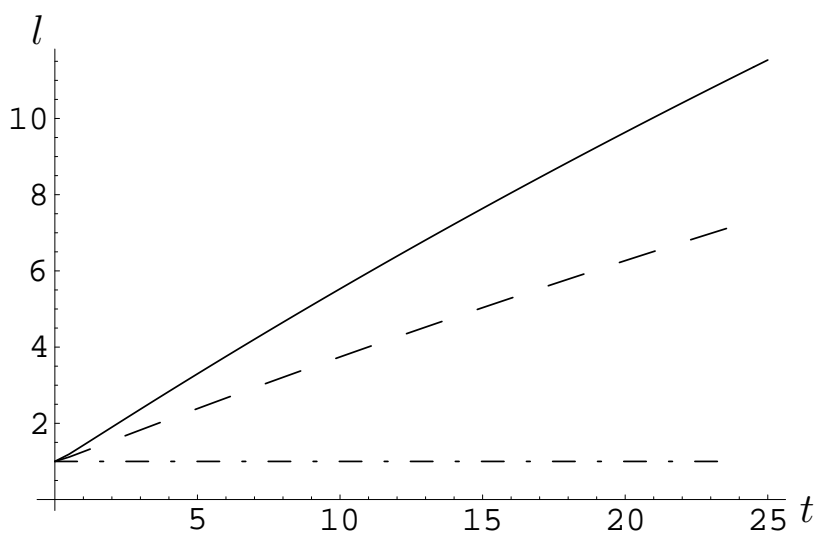

Figure 5. Graph showing how $l$ varies with time, for $k_{2}^{*}=3$ (solid line), $k_{2}^{*}=5$ (dashed line) and $k_{2}^{*}=10$ (dot-dashed line).

remains constant (at $\alpha^{*}$ ). This is because the allowable volume fraction of blood vessels provides insufficient oxygen to sustain the tumour's development.

Finally, we return to consider the behaviour of the time dependent problem defined by equation (85). We investigate the effect that varying $k_{2}^{*}$ and $D$ has on the resulting solutions. In figure 5 we show how changes in the tumour cell death term $k_{2}^{*}$ affect the evolution of the tumour width when $D=1$. As $k_{2}^{*}$ increases, we see that the tumour's growth rate decreases. Further, for $k_{2}^{*}$ sufficiently large, tumour growth cannot occur. In such cases, the internal volume fraction of tumour cells decreases from the natural packing density $\alpha^{*}$ (to its steady state), and hence no stress is generated to facilitate the tumour's expansion. We can determine the maximum value of $k_{2}^{*}$ for which tumour growth occurs by repeating the procedure described earlier for finding $\gamma_{k}$. In this way, we find that

$$
k_{2}^{*} \leq \frac{\gamma_{0}\left(\gamma_{c}+\gamma_{0}\right)\left(1-\alpha^{*}-\gamma_{0}\right)}{\alpha^{*} \gamma^{*}\left(1-\alpha^{*}-\gamma^{*}\right) \gamma_{c}}
$$

is a necessary condition for growth to occur. With $\alpha^{*}=0.6, \gamma_{0}=0.2$ and $\gamma_{c}=0.05$, we find that the tumour will grow if $k_{2}^{*} \leq 8.3$. We note that cellular death alone is insufficient to generate an expanding tumour which has a necrotic region at the centre.

Holding $k_{2}^{*}=3$, in figure 6 we show how the width of the tumour evolves for various $D$. We see that decreasing $D$ decreases the tumour's growth rate. In order to check the small $D$ predictions of $\S 4.1$, we include in figure 7 profiles of $\alpha$ at $t=5.5$ for the three values of $D$ used in figure 6 . 


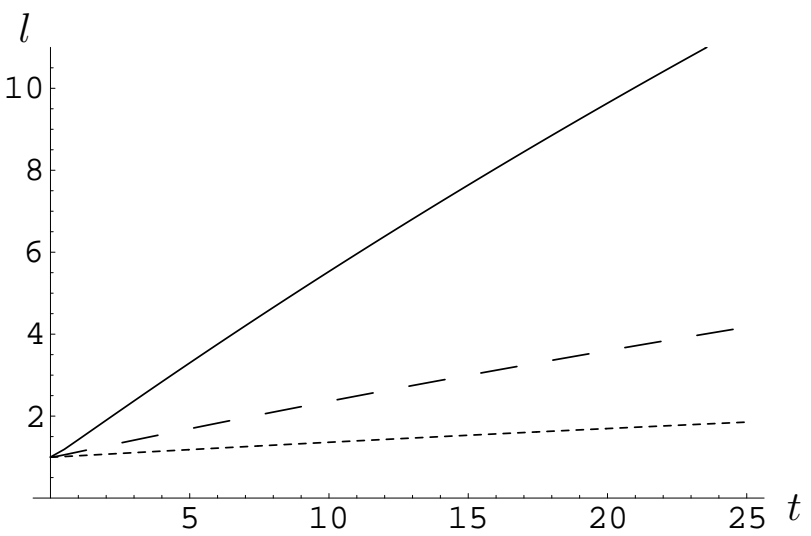

Figure 6. Graph showing how $l$ varies with time, for $D^{*}=1$ (solid line), $D=0.1$ (large-dashed line) and $D=0.01$ (small-dashed line).

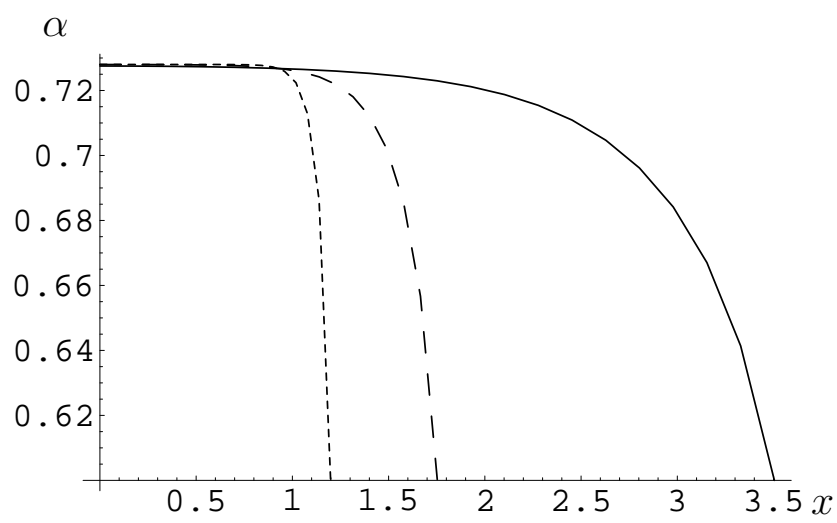

Figure 7. Graph showing how the volume fraction of tumour cells varies with distance from the tumour centre, for $D^{*}=1$ (solid line), $D=0.1$ (large-dashed line) and $D=0.01$ (small-dashed line). Here, $k_{2}^{*}=3$, and we are looking at the instant $t=5.5$.

As predicted in $\S 4.1$, decreasing $D$ decreases the width of the region close to the front in which $\alpha$ increases from $\alpha^{*}$ to its "steady state" value. Physically, this means that the area in which all the spatial variation occurs is confined to a small region (or boundary layer) just inside the free surface.

4.3. The limit $q^{*} \neq 0$. We now allow liquid to enter the tumour from the blood vessels. Setting $\beta_{B}=0.1$ and $k_{5}^{*}=1$ in (61)-(67), we find that there is a slight increase in the tumour's growth rate due to internal supply of mass. Increasing $k_{5}^{*}$ further (to 10 and then to 100) results in a further slight increase in the growth rate (see figure 8). We conclude that allowing internal supply of liquid to the tumour does not, in this instance, significantly enhance the tumour's growth.

4.4. Generation of the necrotic centre. Thus far our vessels have been stiff enough that they have resisted collapse. In this section we consider the effect that the excess pressure has on the neovasculature that is commonly found in tumours. For simplicity, we set $k_{4}^{*}=k_{5}^{*}=k_{6}^{*}=0$. We shall relax this assumption later when we include angiogenesis. We show numerical simulations of the governing equations (61-67) fixing $p_{\text {crit }}=0.3, k_{2}^{*}=3, k_{3}^{*}=5, D=1$ and $\gamma_{c}=0.05$ (with all the other parameters set to zero) in figures 9-14. In figure 9, we see that initially the tumour cells grow throughout the tumour. The build up of internal stress that occurs when the cellular 


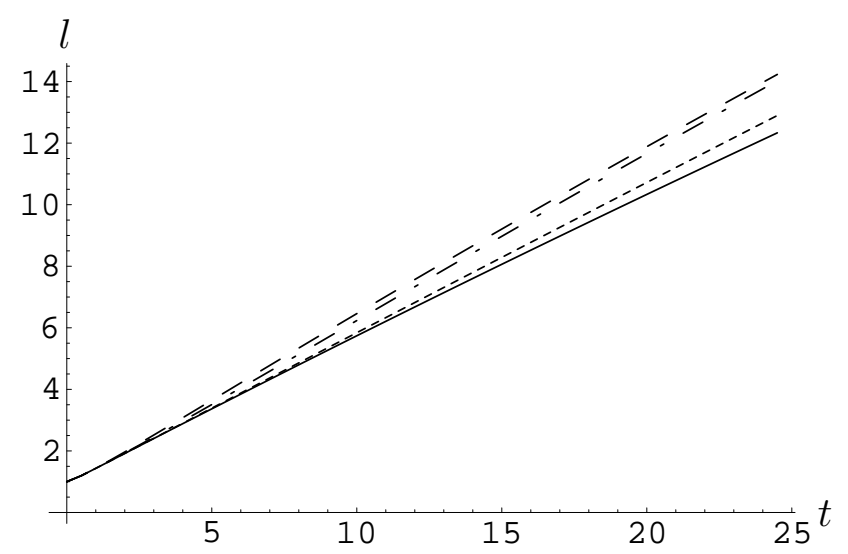

Figure 8. Graph showing how the width of the tumour changes with time, for $k_{5}^{*}=0$ (solid line), $k_{5}^{*}=1$ (small-dashed line), $k_{5}^{*}=10$ (dot-dashed line) and $k_{5}^{*}=100$ (large dashed line).

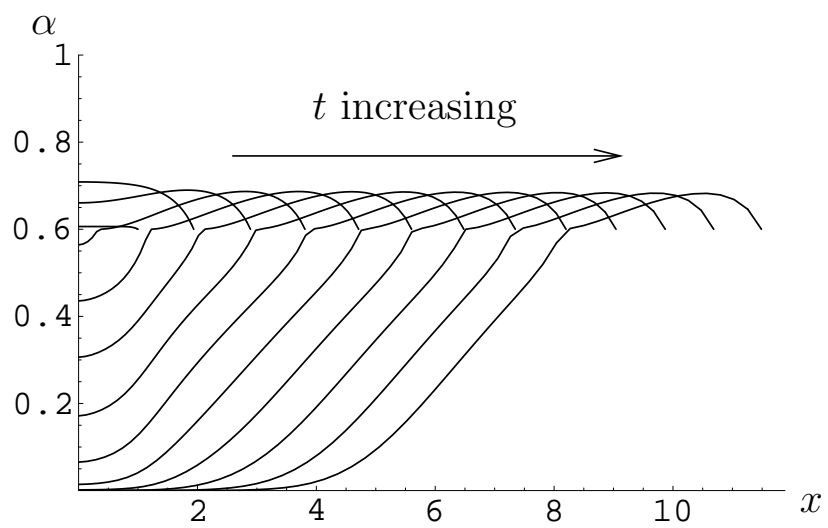

Figure 9. Graph showing how the volume fraction of tumour cells changes through the tumour mass, at $t=0,2,4, \ldots \ldots, 24$.

volume fraction exceeds $\alpha^{*}$ leads to expansion of the tumour mass (see figure 14). The tumour cell pressure increases (see figure 12), and thus the pressure exerted on the blood vessels increases (see figure 13), so that eventually the vessels become occluded and die (see figure 10). Tumour cells in the vicinity of the dying blood vessels also die, and an area of necrosis is formed (see figure 9). Note that we are in a parameter regime for which there is no blood vessel growth. As the tumour cells die, the pressure in this phase decreases. After the initial transient, the system settles to a travelling wave-like profile. From figure 14, we see that the tumour's width increases approximately linearly with time.

From figure 12, we note that the cellular pressure at the tumour centre settles to a steady state level which is lower than that at the tumour front. The tumour cell pressure possesses an internal maximum (greater than the value at the tumour's edge). The pressure in the extracellular material, however, decreases initially, and then develops an internal minumum (see figure 15). The pressure in the extracellular material decreases to this minumum near the tumour front and then increases to a much higher plateau value. We conjecture that it is the difference in the pressure between the plateau region and the minimum (just inside the tumour's edge) that is being referred to in Boucher and Jain (1992), when they state that the interstitial fluid pressure at the centre of the tumour is elevated compared to that at the tumour boundary (effectively, they neglect the small region near the tumour boundary in which the fluid pressure equilibriates with the external pressure). 


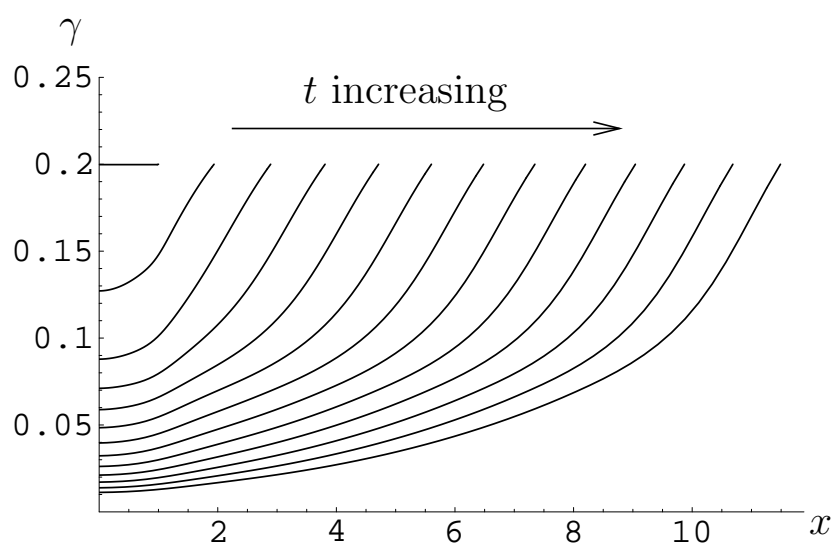

Figure 10. Graph showing how the volume fraction of blood vessels changes through the tumour mass, at $t=0,2,4, \ldots \ldots, 24$.

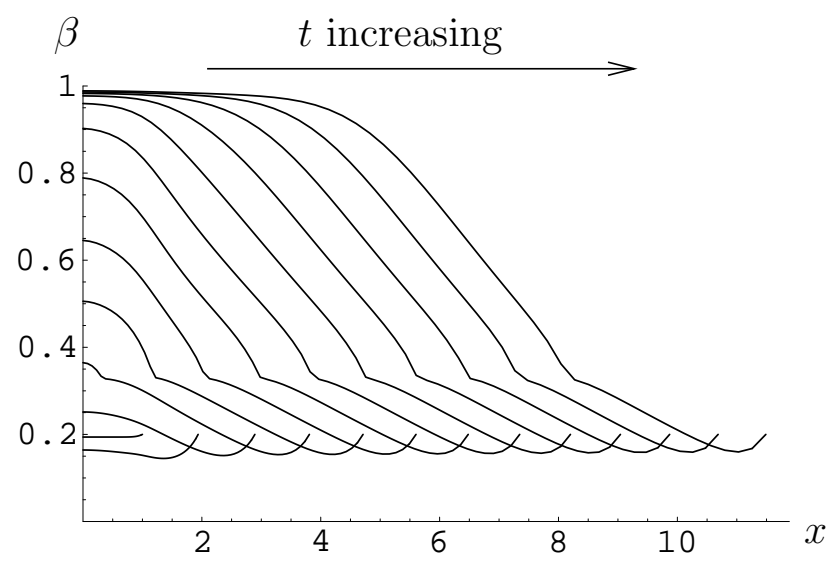

Figure 11. Graph showing how the volume fraction of extracellular material changes through the tumour mass, at $t=0,2,4, \ldots \ldots, 24$.

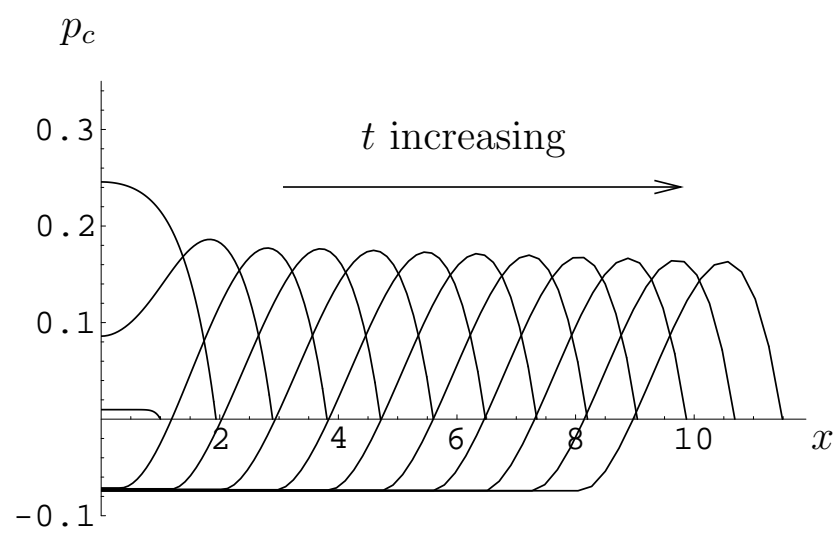

Figure 12. Graph showing how the tumour cell pressure changes through the tumour, at $t=0,2,4, \ldots \ldots$, 24. 


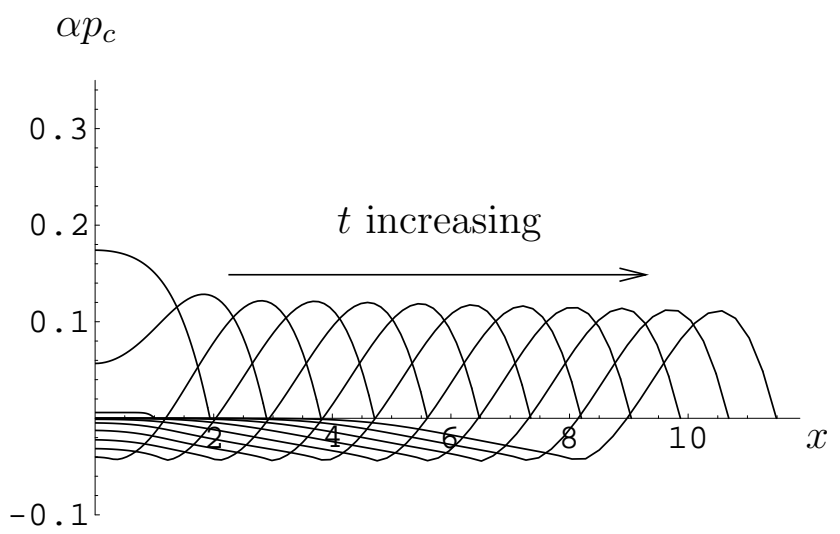

Figure 13. Graph showing how the pressure exerted on the blood vessels, $\alpha p_{c}$, changes through the tumour mass, at $t=0,2,4, \ldots \ldots, 24$.

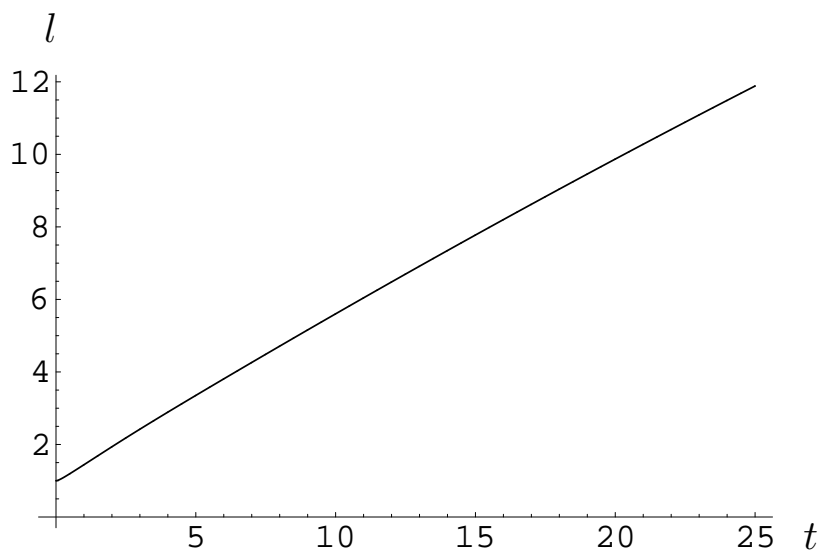

Figure 14. Graph showing how the width of the tumour changes with time

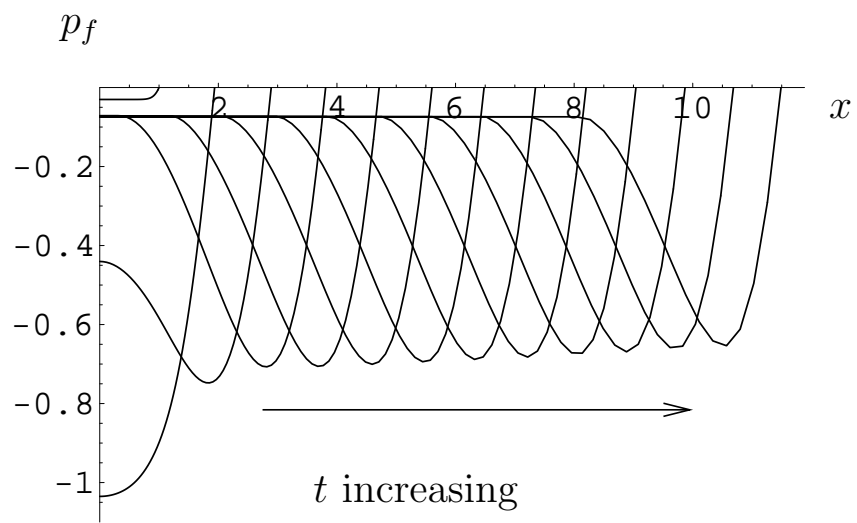

Figure 15. Graph showing how the pressure in the extracellular material changes through the tumour, at $t=0,2,4, \ldots \ldots, 24$. 


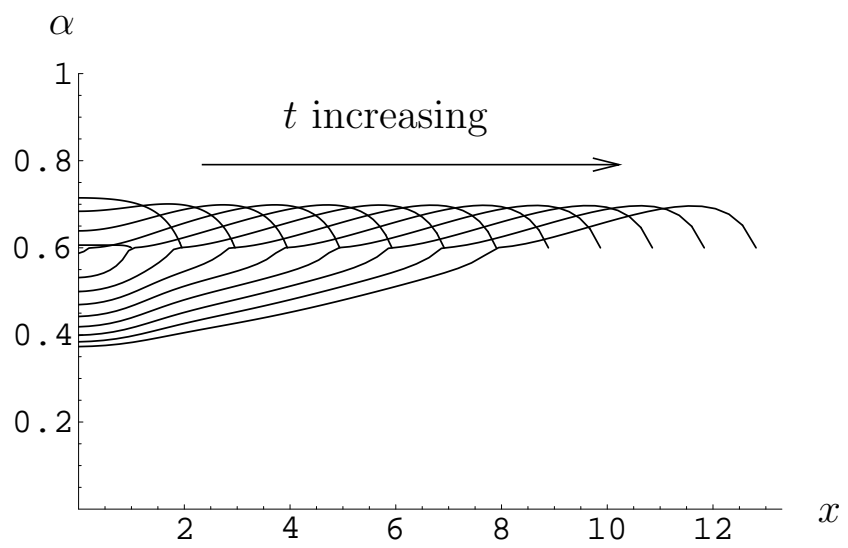

Figure 16. Graph showing how the volume fraction of tumour cells changes across the tumour, at $t=0$, $2,4, \ldots \ldots, 24$.

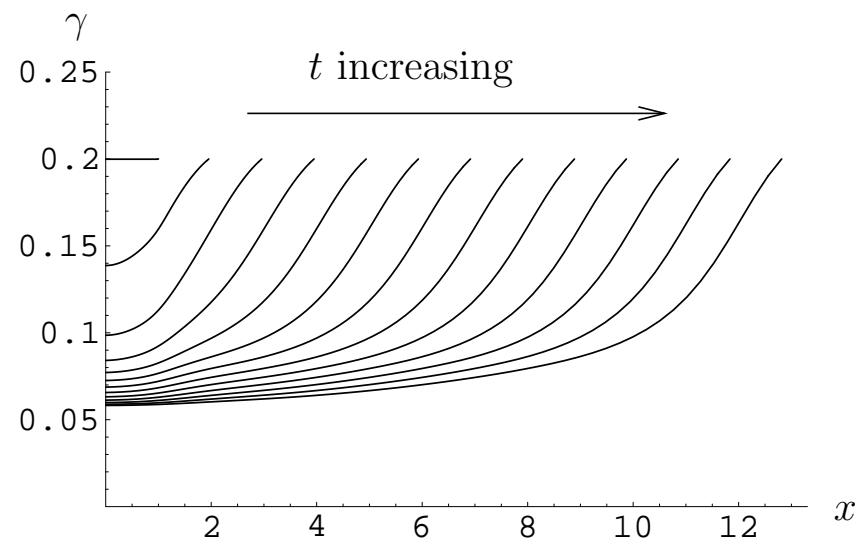

Figure 17. Graph showing how the volume fraction of blood vessels changes across the tumour, at $t=0$, $2,4, \ldots \ldots, 24$.

We now include angiogenesis in our simulations, setting $k_{4}^{*}=0.036$ and $\alpha_{m}^{2}=0.001$ in equations (61)-(67). The resulting plots of the volume fractions of tumour cells, the extracellular , and blood vessels, together with the pressure exerted on the blood vessels are presented in figures 16-19.

Comparing figures 10 and 17, we can see immediately that including angiogenesis into the model increases the volume fraction of blood vessels throughout the tumour (with this set of parameters). Further, the central level of blood vessels is sufficient to keep the tumour cells viable (compare figures 9 and 16). In figure 20, we compare how the tumour width varies with time when angiogenesis is switched off (solid line) and when angiogenesis is switched on (dotted line). As expected, the tumour's growth rate increases when angiogenesis is included. We note that including angiogenesis increases the volume fraction of blood vessels throughout the tumour, not just in regions containing dying tumour cells.

By changing the model parameters we have been able to reproduce spatial structures of vascular tumours described in the introduction. Note, however, that the growth rates do not appear to change much, with the tumour volume increasing approximately cubically with time, as seen in the experiments of Liao et al. (2000) and Candido et al (2001).

4.5. Summary. The model of vascular tumour growth that we have developed in this paper contains a number of different physical mechanisms, each of which influences the details of the 


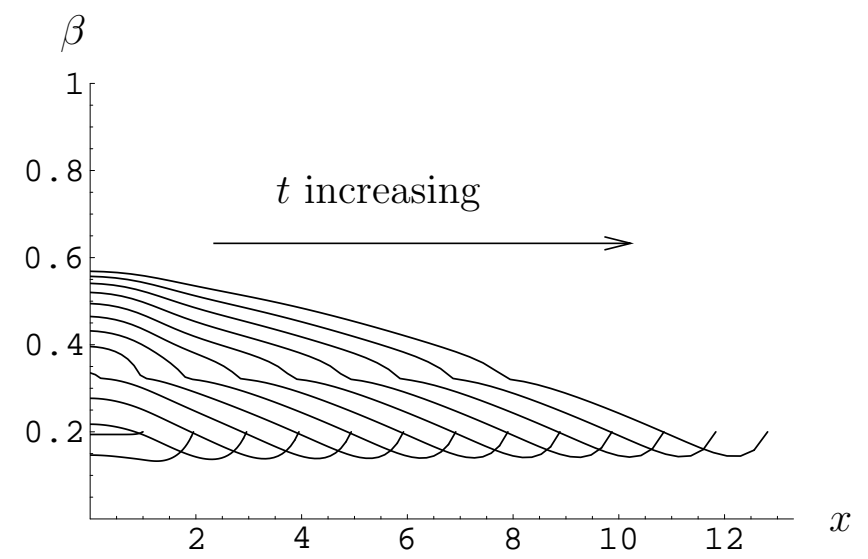

Figure 18. Graph showing how the volume fraction of extracellular material changes across the tumour, at $t=0,2,4, \ldots \ldots, 24$.

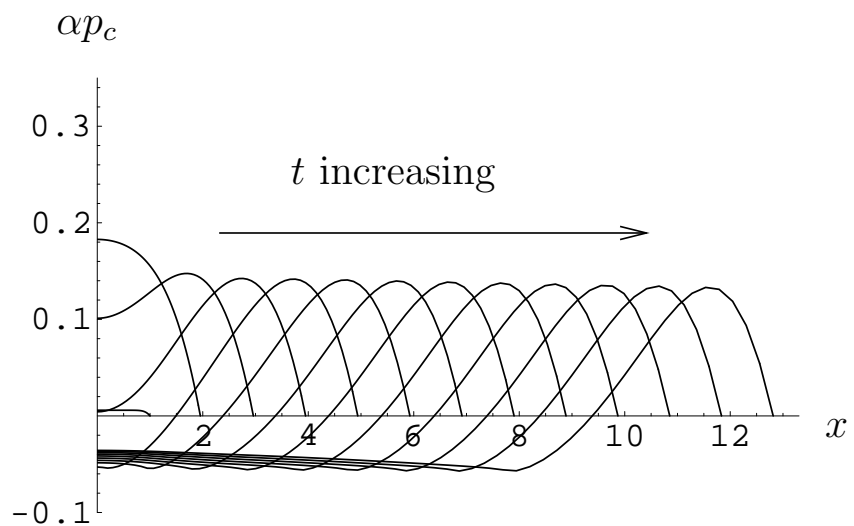

Figure 19. Graph showing how the pressure exerted on the blood vessels changes across the tumour, at $t=0,2,4, \ldots \ldots, 24$.

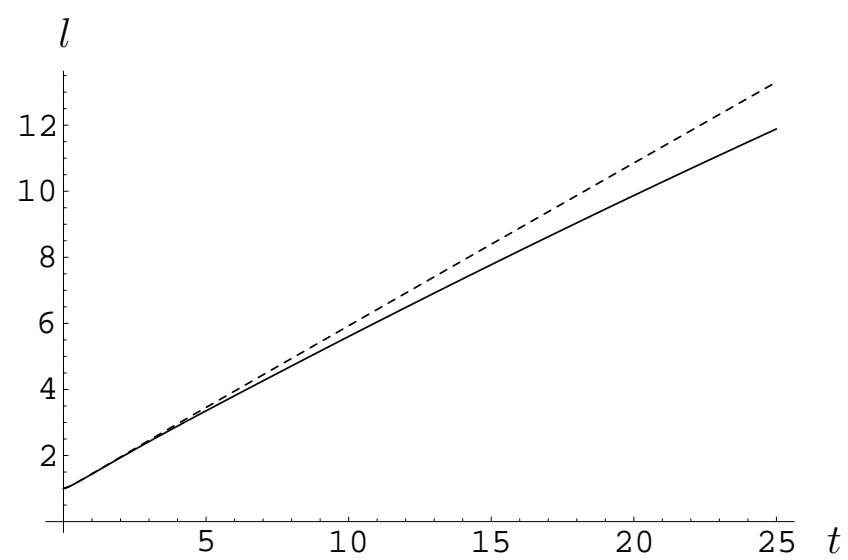

Figure 20. Graph showing how the tumour width changes with time, with angiogenesis switched on (dotted line) compared to no angiogenesis (solid line). 
tumour's development. In this section we have focussed on several mechanisms in order to illustrate the range of behaviours that may arise. For completeness, we present a table that summarises the results obtained in $\S 4.1-\S 4.4$.

\begin{tabular}{|l|l|}
\hline Mechanism & Predicted Growth Pattern \\
\hline $\begin{array}{l}\text { No internal supply or loss of fluid } \\
\text { and no vessel death }\end{array}$ & $\begin{array}{l}\text { Tumour growth resembles avascular growth. } \\
\text { Width increases linearly with time. }\end{array}$ \\
\hline $\begin{array}{l}\text { Fluid delivery to tumour } \\
\text { via blood vessels }\end{array}$ & No qualitative change to the tumour's growth \\
\hline Occlusion of blood vessels & $\begin{array}{l}\text { Pressure on blood vessels causes their collapse. } \\
\text { Blood vessel death creates necrotic centre. } \\
\text { Pressure in extracellular phase has internal minimum } \\
\text { and rises to a plateau value in the centre. }\end{array}$ \\
\hline Angiogenesis & $\begin{array}{l}\text { Volume fraction of tumour cells and blood vessels } \\
\text { increases throughout the tumour, } \\
\text { whose growth rate increases. }\end{array}$ \\
\hline
\end{tabular}

\section{Discussion}

In this paper our aim was to develop a modelling framework for studying vascular tumour growth. One of our main assumptions in developing the model was that the blood vessels in the tumour would collapse if the pressure exerted on the vessels by the tumour cells exceeded a critical value. Our model was based on a multiphase approach in which conservation of mass and momentum equations were formulated for each of the three phases: tumour cells, blood vessels and extracellular material. We simplified the resulting system of equations and boundary conditions, obtaining the parabolic equation (30) and the hyperbolic equation (31) describing the evolution of the volume fractions of the tumour cell and blood vessel phases. The former equation is similar to that used by other authors as a starting point for modelling tumour growth (Gatenby and Gawlinski (1996), Sherratt (2000)). We illustrated one possible growth regime by setting all the drag coefficients equal. In this regime, we found that, when the drag coefficients were all small, the volume fraction of tumour cells changed rapidly in a boundary layer close to the free surface, see figure 7 . In the case where there was no birth or death of blood vessels, and no internal sources or sinks of water, the blood vessel density was found to be a step function changing from $\gamma=\gamma_{0}$ (the initial volume fraction of blood vessels) to $\gamma=\gamma^{*}$ (the external volume fraction of blood vessels) at the depth in the tumour associated with the tumour's initial width, i.e. the tumour grew around and co-opted the external blood vessels. We found relationships for determining whether a tumour in this parameter limit would grow.

When we allowed liquid to enter the tumour from the vasculature, we saw that it did not significantly affect the rate at which the tumour grew (see figure 8).

We then examined the effect of including blood vessel collapse. We saw (figures 9 and 10) that an area of of cellular necrosis was generated due to a local collapse in blood vessels. We also saw that the pressure in the extracellular material had a constant value at the centre of the tumour (figure 15), then decreased to a minumum a short distance from the edge (before rising to the tissue pressure at the edge). We commented that the difference between the central pressure and the pressure at the minimum has probably been observed experimentally in Boucher and Jain (1992). The tumour width increased linearly with time (figure 14), indicating a cubic growth rate for the tumour volume, which is consistent with the experimental evidence given in the introduction (see 
Candido et al (2001); Liao et al. (2000)). We introduced angiogenesis into the model and saw that the volume fraction of both tumour cells and blood vessels increased throughout the tumour (figures 16 and 17). Introducing angiogenesis increased the rate of growth of the tumour.

Throughout the paper, our philosophy when modelling has been to use the simplest functional forms that capture the physical phenomena that we are aiming to describe. By modifying these terms it should be possible to tailor the model to describe specific tumours. For example, we could alter the tumour cell growth rate term so that it levels off when the blood vessel density is high. We do not anticipate that such modifications will alter the qualitative behaviour of the model. We have also used a one-dimensional cartesian model for simplicity. We expect the radially-symmetric analogue of our model to have qualitatively the same internal structure, but that the tumour growth rate may be altered by the change in geometry.

We note also that our model does not account for interactions with the tissue surrounding the tumour. Following Chen et al. (2001), we could address this issue by including a description of the mechanical properties of the external tissue and modifying our boundary conditions to take account of the deformation of the external tissue. Our model also does not take into account the behaviour of dead tumour cells, nor the rigidity provided by the extracellular matrix. These effects could be incorporated by introducing extra phases into the model, which would increase its complexity. We expect that a dead cell phase would exert pressure on the blood vessel phase in addition to that exerted by the live cells, which would result in further vessel death.

In our opinion the mechanism that needs to be considered in more detail is the constitutive law used to describe the growth of new blood vessels. In this paper the angiogenesis term that we employ leads simply to an increase in the volume fraction of blood vessels throughout the tumour. We should modify this term to capture more accurately the observation that angiogenesis is usually more pronounced in regions where there is substantial cell death and/or hypoxia. Two alternative modelling strategies that could be used to address this issue involve (i) making the rate of blood vessel formation $k_{4}$ depend on the rate of change of the volume fraction of tumour cells $(\partial \alpha / \partial t)$ and (ii) introducing oxygen as a dependent variable which is supplied to the tumour via the vasculature and to the tumour by diffusion and making $k_{4}$ increase as the oxygen tension decreases. As mentioned in the introduction, as the new vessels age they develop a basement membrane and become more resistant to collapse. To capture this process, we could let the "critical pressure" for vessel collapse be a known function of position and time. In this case, we would be intrinsically saying that we knew which bits of the vasculature were new and which were old. A better model might be to include two populations of blood vessels, neovasculature and mature vessels, each with different properties. The neovasculature would be born by angiogenesis and prone to collapse. The mature vessels would be co-opted from the host's vasculature and would also be formed by maturing neo-vessels. These vessels would be less prone to collapse. We plan to investigate all these alternatives in future work.

A further issue that we plan to investigate involves using the model to study the efficacy of different anti-angiogenic drugs (such as angiostatin (O'Reilly et al., 1994) and endostatin (O'Reilly et al., 1997)), and vascular targetting agents (such as combretastatin (Galbraith et al., 2001)). By introducing new dependent variables to describe the drug concentration in the vessels and in the tissue itself and modifying the angiogenesis and vessel death terms to account for the ways in which the different drugs are believed to act, it should be possible to predict which types of tumours will respond better to which anti-angiogenic (or anti-vascular) therapies.

With suitable choices for the parameters, our model is able to capture the behaviour of tumours with several different spatial compositions. For example, by excluding internal sources and sinks of mass, and blood vessel death, we are able to describe growth in which the vascular density is constant, and the growth resembles avascular growth. Including pressure occlusion of vessels 
enables us to generate tumours which have qualitatively the same structure as the murine tumours described in the introduction, namely a vascular tumour in which there is a central region of necrosis and a healthy area surrounding this in which blood vessels provide the oxygen and water that the tumour cells need to survive and proliferate. The final point to note is that the growth rate of the tumour increased when we included angiogenesis, as compared to the case when angiogenesis was switched off.

In conclusion, we have developed a mathematical model describing vascular tumour growth that incorporates details of the interactions between the tumour cells and the developing vasculature. In its present form, the model is still general, although it should be relatively straightforward to tailor it to describe specific tumour cell lines and their responses to different anti-angiogenic and anti-vascular therapies.

\section{Acknowledgements}

CJWB wishes to acknowledge that part of his work was carried out with the financial assistance from EPSRC in the form of a ROPA award (to CEL and HMB). HMB wishes to acknowledge financial support from EPSRC in the form of an Advanced Fellowship.

\section{References}

Baish, J. W., Y. Gazit, D. A. Berk, M. Nozue, L. T. Baxter and R. K. Jain(1996). Role of tumour vasculature architecture in nutrient and drug delivery: an invasion percolation-based network model. Microvasc. Res., 51 (3), 327-346.

Beliën, J. A. M., P. J. Van Diest and J. P. A. BaAK (1999). Relationships between vascularization and proliferation in invasive breast cancer. J. Pathol., 189, 309-318.

Bicknell, R., C. E. Lewis And N. Ferrara (1997) Tumour Angiogenesis. Oxford University Press, Oxford.

Boucher, Y., AND R. K. JAIN (1992). Microvascular pressure is the principal driving force for interstitial hypertension in solid tumours: implications for vascular collapse. Cancer Res., 52, 5110-5114.

Breward, C. J. W., H. M. Byrne and C. E. Lewis (2001). Modeling the interactions between tumour cells and a blood vessel in microenvironment within a vascular tumour. Euro. J. Appl. Math., 12, 529-556.

Breward, C. J. W., H. M. Byrne And C. E. Lewis (2002). The role of cell-cell interactions in a two-phase model for avascular tumour growth. J. Math. Biol., 45, 125-152.

Brown, N. J., C. A. Staton, G. R. Rodgers, K. P. Corke, J. C. E. Underwood and C. E. LEwis (2002). Fibrinogen E fragment selectively disrupts the vasculature and inhibits the growth of tumours ina syngeneic murine model. Brit. J. Cancer, 86: (11), 1813-1816.

Byrne, H. M. and M. A. J. Chaplain (1995). Growth of nonnecrotic tumours in the presence and absence of inhibitors Math. Biosci., 2, 151-181.

Candido, K. A., K. Shimizu, J. C. Mclaughlin, R. Kunkel, J. A. Fuller, B. G. Redman, E. K. Thomas, B. J. Nickoloff And J. J. Mule (2001). Local administration of dendritic cells inhibits established breast tumour growth: implications for apoptosis-inducing agents. Cancer Res., 61, 228-236.

Chen, Y-.C., H. M. Byrne And J. R. King (2001). The influence of growth-induced stress from the surrounding medium on the development of multicell spheroids. J. Math. Biol., 43, 191-220. 
Galbraith, S. M., D. J. Chaplin, F. Lee, M. R. L. Stratford, R. J. Locke, B. Vojnovic, G. M. TOzer (2001). Effects of combretastatin A4 phosphate on endothelial cell morphology in vitro and relationship to tumour vascular targeting activity in vivo. Anticancer Res., 21:(1A), 93-102.

Gatenby, R. A. and E. T. Gawlinski (1996). A reaction-diffusion model of cancer invasion. Cancer Res., 56, 5745-5753.

Griffon-Etienne, G., Y. Boucher, C. Brekken, H. D. Suit and R. K. Jain (1999). Taxane-induced apoptosis decompressed blood vessels and lowers interstitial fluid pressure in solid tumors: clinical implications. Cancer Res., 59, 3776-3782.

Hahnfield, P., D. Panigraphy, J. Folkman and L. Hlatky (1999). Tumour development under angiogenic signalling: a dynamic theory of tumour growth, treatment response and postvascular dormancy. Cancer Res., 59, 4770-4775.

Hashizume, H., P. Baluk, S. Morikawa, J. W. Mclean, G. Thurston, S. Roberge, R. K. Jain And D. M. MCDonald (2000). Openings between defective endothelial cells explain tumor vessel leakiness. Am. J. Pathol., 156:4, 1363-1380.

Kozin, S. V., Y. Boucher, D. J. Hicklin, P. Bohlen, R. K. Jain and H. D. Suit (2001). Vascular endothelial growth factor receptor-2-blocking antibody potentiates radiation-induced long-term control of human tumour xenografts Cancer Res., 61, 39-44.

Krogh, A. (1919). The number and distribution of capillaries in muscles with calculations of the oxygen pressure head necessary for supplying the tissue. J. Physiol., 52, 409-415.

Liao F., Y. Li, W. O'Connor, L. Zanetta, R. Bassi, A. Santiago, J. Overholser, A. Hooper, P. Minatti, E. Dejana, D. J. Hicklin and P. Bohlen (2000). Monoclonal antibody to vascular endothelial-chadherin is a potent inhibitor of angiogenesis, tumour growth and metastasis Cancer Res., 60, 6805-6810.

FowleR, A. C. (1997). Mathematical models in the applied sciences. Cambridge University Press, Cambridge.

Jackson, T. L. And H. M. Byrne (2000). A mathematical model to study the effects of drug resistance and vasculature on the response of solid tumors to chemotherapy Math. Biosci., 164, $17-38$.

Maseide, K. And E. K. Rofstad (2000). Mathematical modelling of chronic hypoxia in tumours considering potential doubling time and hypoxic cell lifetime Radiother. Oncol., 54, 171-177.

O’Reilly, M. S., L. Holmgren, Y. Shing, C. Chen, R. A. Rodenthal, M. Moses, W. S. Lane, Y. Cao, E. H. Sage and J. Folkman (1994). Angiostatin. A novel angiogenesis inhibitor that mediates the suppression of metastases by a Lewis lung carcinoma. Cell, $\mathbf{7 9}, 315-$ 328.

O’Reilly, T. Boehm, Y. Shing, N. Fukai, G. Vasios, W. S. Lane, E. Flynn, J. R. Birkhead, B. R. Olsen And J. Folkman (1997). Endostatin. An endogenous inhibitor of angiogenesis and tumour growth. Cell, 88, 277-285.

Orme, M. E. And M. A. J. Chaplain (1996). A mathematical model of vascular tumour growth and invasion. Math. Comp. Mod., 23:10, 43-60.

Sherratt, J. A. (2000). Wave front propagation in a competition equation with a new motility term modelling contact inhibition between cell populations Proc. Roy. Soc. Lond. A, 456, 23652386 .

Todo, T., R. L. Martuza, M. J. Dallman and S. D. Rabkin (2001). In Situ expression of soluble B7-1 in the context of oncolytic herpes simplex virus induces potent antitumor immunity Cancer Res., 61, 153-161.

Towle, M. J., K. A. Salvato, J. Budrow, B. F. Wels, G. Kuznetsov, K. K. Aalfs, S. Welsh, W. Zheng, B. M. Seletsky, M. H. Palme, G. J. Habgood, L. A. Singer, L. 
V. DiPietro, Y. Wany, J. J. Chen, D. A. Quincy, A. Davis, K. Yoshimatsu, Y. Kishi, M. J. Yu AND B. A. LitTlefield (2001). In vitro and in vivo anticancer activities of synthetic macrocyclic ketone analogues of halichondrin B Cancer Res., 61, 1013-1021.

WARD, J. P. AND J. R. KING (1997). Mathematical modelling of avascular tumour growth. IMA J. Math. Appl. Med., 14, 39-69. 